\title{
Value Distribution of Holomorphic Curves on an Angular Domain
}

\author{
ZHENG JIAN-HUA
}

\begin{abstract}
In this paper, we investigate the value distribution of holomorphic curves on an angular domain from the point of view of potential theory and establish the first and second fundamental theorems corresponding to those theorems of Ahlfors-Shimizu, Nevanlinna, and Tsuji on meromorphic functions in an angular domain, which have not been established before in other references. As applications of these theorems, we introduce the singular directions of holomorphic curves and prove their existences and investigate the growth of holomorphic curves with radially distributed hyperplanes and uniqueness of holomorphic curves in an angular domain. The obtained results are transferred to the algebroid functions.
\end{abstract}

\section{Introduction}

For $0 \leq \alpha<\beta \leq 2 \pi$, by $\Omega(\alpha, \beta)$ we denote the angular domain

$$
\Omega(\alpha, \beta):=\{z: \alpha<\arg z<\beta\}
$$

and by $\bar{\Omega}(\alpha, \beta)$ its closure. Sometimes, without occurrence of any confusion in the context, we write simply $\Omega$ instead of $\Omega(\alpha, \beta)$. The behavior of a function meromorphic in an angle has been investigated in many references, such as [23; $2 ; 15 ; 4]$, and [31]. For the purpose of studying the topic, characteristic functions that describe the growth of the meromorphic functions in an angle have been introduced; for instance, the Ahlfors-Shimizu characteristic, Nevanlinna characteristic, and Tsuji characteristic for an angle, and the corresponding main theorems have been established. The second main theorem for the Ahlfors-Shimizu characteristic for an angle can be found in [29], and in [12] for the Nevanlinna characteristic and Tsuji characteristic for an angle. These main theorems were collected and compared to each other in [31].

We emphasize the usage of the Ahlfors-Shimizu second main theorem for an angle in proving the existence of $T$-direction of a meromorphic function on the complex plane; see [31] for a detailed discussion on singular directions. We remark that $\Omega_{\varepsilon}=\Omega(\alpha+\varepsilon, \beta-\varepsilon)$ in the theorem cannot be replaced by $\Omega$.

The Nevanlinna second main theorem for an angle was used in $[12 ; 10 ; 26 ; 30]$, and [31] to investigate the growth of meromorphic functions with some radially distributed values. The usage of the second main theorem produces a basic and

Received December 10, 2014. Revision received January 22, 2015.

The work is supported by NSF of China (No. 11171170). 
elementary method in the topic [30]. In [31], in view of the Tsuji second main theorem, we established a five-value uniqueness theorem and four-value uniqueness theorem for meromorphic functions in an angle.

These results motivate us to consider the case of holomorphic curves. There seems to be few corresponding investigations of holomorphic curves in comparison to those of meromorphic functions; for instance, do we have an analogue of the Ahlfors-Shimizu second main theorem for an angle for holomorphic curves in an angle? In this paper, we confirm the question and establish the second main theorems for holomorphic curves in an angle. We stress that we could not obtain the Ahlfors-Shimizu second main theorem for a holomorphic curve in an angle in terms of the Ahlfors theory of covering surfaces as in the case of meromorphic functions. The potential theory of Eremenko and Sodin [8] in theory of value distribution is powerful in discussion of this topic. We will attain our purpose by using their exposition from the point of view of potential theory. As an application of the second main theorem, we give a definition of singular directions of holomorphic curves and prove their existence. There are few results on the singular directions of holomorphic curves; so far, we know that the Julia direction was studied in Eremenko [6] in view of circles de remplissage and Tu [24] in view of the normality criteria of holomorphic curve family. Therefore, this seems to be a new topic in essence.

As applications of the Nevanlinna and Tsuji second main theorems for an angle, we will discuss the growth of holomorphic curves with radially distributed hyperplanes and $\mathrm{ABC}$ problem, Fermat-type equation, Waring problem, and uniqueness theorems of holomorphic curves in an angle instead of the whole complex plane.

Finally, the obtained results for holomorphic curves are transferred to the case of algebroid functions.

\section{Characteristics of Holomorphic Curves in an Angle}

Let $\mathcal{P}^{n}(\mathbb{C})$ be the $n$-dimensional complex projective space, that is, $\mathcal{P}^{n}(\mathbb{C})=$ $\mathbb{C}^{n+1} \backslash\{0\} / \sim$, where $\sim$ is the equivalence relation defined by that $\left(a_{0}, a_{1}, \ldots\right.$, $\left.a_{n}\right) \sim\left(b_{0}, b_{1}, \ldots, b_{n}\right)$ if and only if $\left(a_{0}, a_{1}, \ldots, a_{n}\right)=\lambda\left(b_{0}, b_{1}, \ldots, b_{n}\right)$ for some $\lambda \in \mathbb{C} \backslash\{0\}$. We denote the equivalence class of $\left(a_{0}, a_{1}, \ldots, a_{n}\right)$ by $\left[a_{0}: a_{1}\right.$ : $\left.\ldots: a_{n}\right]$.

Let $\Omega$ be a domain on $\mathbb{C}$. A map $f: \Omega \rightarrow \mathcal{P}^{n}(\mathbb{C})$ is called a holomorphic curve on $\Omega$ if we can write $f=\left[f_{0}: f_{1}: \ldots: f_{n}\right]$ where all $f_{j}$ are complex analytic functions on $\Omega$ that have no common zeros on $\Omega ; \mathbf{f}=\left(f_{0}, f_{1}, \ldots, f_{n}\right)$ is called a reduced representation of $f$. Set

$$
v(z)=\bigvee_{j=0}^{n} \log \left|f_{j}(z)\right|, \quad z \in \Omega .
$$

Obviously, $v(z)$ is subharmonic on $\Omega$. Let $\mu$ be the Riesz measure of $v(z)$. In fact, $\mu=\frac{1}{2 \pi} \Delta v$, where $\Delta$ is the Laplacian. Let $B\left(z_{0}, r\right)=\left\{z:\left|z-z_{0}\right|<r\right\}$ and 
$B(r)=\{z:|z|<r\}$ throughout the paper. Define

$$
\mathcal{A}(r, \Omega, f)=\mu(\overline{B(r) \cap \Omega})
$$

and

$$
\mathcal{T}(r, \Omega, f)=\int_{0}^{r} \frac{\mathcal{A}(t, \Omega, f)}{t} \mathrm{~d} t .
$$

The quantity $\mathcal{A}(r, \Omega, f)$ is called the unintegrated Ahlfors-Shimizu characteristic of a holomorphic curve $f$ on $\Omega$, and $\mathcal{T}(r, \Omega, f)$ is called the Ahlfors-Shimizu characteristic of a holomorphic curve $f$ on $\Omega$.

A hyperplane $H$ in $\mathcal{P}^{n}(\mathbb{C})$ is

$$
H=\left\{\left[x_{0}: x_{1}: \ldots: x_{n}\right]: \sum_{k=0}^{n} a_{k} x_{k}=0\right\} \text {, }
$$

where $\left(a_{0}, a_{1}, \ldots, a_{n}\right) \in \mathbb{C}^{n+1} \backslash\{0\}$. Obviously, $H$ is completely determined by $\left[a_{0}: a_{1}: \ldots: a_{n}\right]$. Sometimes, we call a nonzero vector $\mathbf{a}=\left(a_{0}, a_{1}, \ldots, a_{n}\right)$ a hyperplane.

Define

$$
\langle\mathbf{f}(z), \mathbf{a}\rangle=a_{0} f_{0}(z)+a_{1} f_{1}(z)+\cdots+a_{n} f_{n}(z) ;
$$

sometimes, to simplify the formula, we write $w_{\mathbf{a}}(z)=\langle\mathbf{f}(z), \mathbf{a}\rangle$. If $\langle\mathbf{f}(z), \mathbf{a}\rangle \not \equiv 0$, we say that $\mathbf{f}$ and $\mathbf{a}$ are free, and we further always assume that they are free when $\langle\mathbf{f}(z), \mathbf{a}\rangle$ appears in the context. Write $v_{\mathbf{a}}(z)=\log |\langle\mathbf{f}(z), \mathbf{a}\rangle| ; v_{\mathbf{a}}$ is subharmonic on $\Omega$. By $\mu_{\mathbf{a}}$ we denote the Riesz measure of $v_{\mathbf{a}}$. Set

$$
n_{\Omega}(r, \mathbf{a}, f)=\mu_{\mathbf{a}}(\overline{B(r) \cap \Omega}),
$$

and hence $n_{\Omega}(r, \mathbf{a}, f)$ is the number of zeros of $\langle\mathbf{f}(z), \mathbf{a}\rangle$ in $B(r) \cap \Omega$. Define

$$
N_{\Omega}(r, \mathbf{a}, f)=\int_{1}^{r} \frac{n_{\Omega}(t, \mathbf{a}, f)}{t} \mathrm{~d} t .
$$

Let $\mathbf{a}_{1}, \mathbf{a}_{2}, \ldots, \mathbf{a}_{q}$ be $q$ hyperplanes. The hyperplanes $\mathbf{a}_{1}, \mathbf{a}_{2}, \ldots, \mathbf{a}_{q}$ are said to be in $s$-subgeneral position if for any $1 \leq i_{0}<i_{1}<\cdots<i_{s} \leq q$, the linear span of $\mathbf{a}_{i_{0}}, \mathbf{a}_{i_{1}}, \ldots, \mathbf{a}_{i_{s}}$ is $\mathbb{C}^{n+1}$, to be in $s$-general position if every $s+1$ members of $\mathbf{a}_{1}, \mathbf{a}_{2}, \ldots, \mathbf{a}_{q}$ are linearly independent and the linear span of $\mathbf{a}_{1}, \mathbf{a}_{2}, \ldots, \mathbf{a}_{q}$ has dimension $s+1$, and to be in general position if $s=n$, that is, if no $n+1$ members of $\mathbf{a}_{1}, \mathbf{a}_{2}, \ldots, \mathbf{a}_{q}$ are linearly dependent.

TheOREM 2.1. Let $\Omega=\{z: \alpha<\arg z<\beta\}$, and let $f$ be a holomorphic curve on $\bar{\Omega}$. Let $\mathbf{a}_{1}, \mathbf{a}_{2}, \ldots, \mathbf{a}_{q}$ be $q$ hyperplanes in general position. Then for arbitrary $\epsilon>0$ such that $\alpha+\epsilon<\beta-\epsilon$, we have

$$
(q-2 n) \mathcal{A}\left(r, \Omega_{\epsilon}, f\right) \leq \sum_{k=1}^{q} n_{\Omega}\left(r, \mathbf{a}_{k}, f\right)+o(\mathcal{A}(r, \Omega, f))+O(\log r)
$$

and

$$
(q-2 n) \mathcal{T}\left(r, \Omega_{\epsilon}, f\right) \leq \sum_{k=1}^{q} N_{\Omega}\left(r, \mathbf{a}_{k}, f\right)+o(\mathcal{T}(r, \Omega, f))+O(\log r)^{2}
$$


for $r \notin E$, where $\Omega_{\epsilon}=\{z: \alpha+\epsilon<\arg z<\beta-\epsilon\}$, and $E$ has a finite logarithmic measure, that is, $\int_{E} \frac{\mathrm{d} t}{t}<\infty$.

We remark that since $E$ is determined by $f$, we often write it as $E(f)$. Eremenko and Sodin [8] proved (2.1) and (2.2) for $\Omega=\Omega_{\epsilon}=\mathbb{C}$ without $\log r$ and $(\log r)^{2}$ in view of their potential theory. Their method is very important. We emphasize that they published a series of papers (see [7] for references) to form their potential theory in theory of value distribution. We use their arguments in [8] to complete the proof of Theorem 2.1.

From Lemmas 6 and 7 in [8] we have the following:

Lemma 2.1. Let $r>0,0<\Gamma<\frac{1}{2} r, r^{\prime}=r+\Gamma$, and let $\Omega=\Omega(\alpha, \beta)$ and $\Omega_{\epsilon}=$ $\Omega(\alpha+\epsilon, \beta-\epsilon)$ for $0<\epsilon<\frac{\beta-\alpha}{2}$. Then there exist three absolute constants $c_{1}$, $c_{2}, c_{3}$ and finitely many disks $B\left(z_{j}, \rho_{j}\right) \subset \Omega\left(r^{\prime}\right), 1 \leq j \leq p$, such that

(i) $p \leq c_{1}\left(\frac{r}{\Gamma}+\log r\right)$;

(ii) every point $z \in \Omega\left(r^{\prime}\right)$ belongs to at most $c_{2}$ disks $B\left(z_{j}, \rho_{j}\right)$, and $\left\{B\left(z_{j}, \rho_{j}\right)\right\}$ covers $\Omega_{\epsilon}(r)$

(iii) there exist nonnegative infinitely differential functions $\varphi_{j}$ with $\operatorname{supp} \varphi_{j} \subset$ $B\left(z_{j}, \rho_{j}\right)$ such that the function

$$
\Phi(z)=\sum_{j=1}^{p} \varphi_{j}(z)
$$

satisfies

$$
0 \leq \Phi(z) \leq 1 \quad \text { and }, \quad \text { on } \Omega_{\epsilon}(r), \quad \Phi(z)=1,
$$

and letting $\psi_{j}(z)=\varphi_{j}\left(\rho_{j} z+z_{j}\right)$, we have

$$
\left|\operatorname{grad} \psi_{j}(z)\right| \leq c_{3} ;
$$

(iv) Let $\mu, v$ be two nonnegative finite measures on $\Omega(r)$. Set

$$
\begin{aligned}
a_{j} & =\int \varphi_{j} \mathrm{~d} \mu, \\
J_{K} & =\left\{j: 1 \leq j \leq p, a_{j} \leq K\right\}, \\
J_{v, \gamma} & =\left\{j: 1 \leq j \leq p, \gamma a_{j} \leq v\left(B\left(z_{j}, \rho_{j}\right)\right)\right\} .
\end{aligned}
$$

We have

$$
\begin{aligned}
\sum_{j \in J_{K}} a_{j} & \leq K p, \\
\sum_{j \in J_{v, \gamma}} a_{j} & \leq \frac{c_{2}}{\gamma} \nu\left(\Omega\left(r^{\prime}\right)\right) .
\end{aligned}
$$

Now we are in the position to prove Theorem 2.1. 
Proof. We use the notation in the proof of Theorem 1 of Eremenko and Sodin [8] with the same meanings, for instance,

$$
\mathcal{A}(r)=\mathcal{A}(r, \Omega, f), \quad r^{\prime}=r+\Gamma, \quad \Gamma=\frac{r}{\log ^{\eta} \mathcal{A}(r)}, \quad \eta>1, \varepsilon>0,
$$

and $\mathcal{A}\left(r^{\prime}\right) \leq(1+\varepsilon) \mathcal{A}(r)$ for $r \notin E$, where $E$ is a subset of $[0,+\infty)$ of finite logarithmic measure. We assume without loss of generality that

$$
\frac{\mathcal{A}(r)}{\log r} \rightarrow \infty \quad(r \rightarrow \infty) .
$$

Set

$$
\Lambda(r)=\frac{\mathcal{A}(r)}{\log r}
$$

and

$$
\begin{gathered}
K(r)=\frac{\mathcal{A}(r)}{\max \left\{\sqrt{\Lambda(r)} \log r, \log ^{\tau} \mathcal{A}(r)\right\}}, \quad \tau=\eta^{2}, \gamma=\frac{1}{\varepsilon}, \\
\nu=\mu+\sum_{k=1}^{q} \mu_{k},
\end{gathered}
$$

where $\mu=\frac{1}{2 \pi} \Delta v$ and $\mu_{k}=\frac{1}{2 \pi} \Delta v_{k}, v_{k}=\log \left|\left\langle\mathbf{f}, \mathbf{a}_{k}\right\rangle\right|$. Obviously, $K(r) \rightarrow \infty$ as $r \rightarrow \infty$. Then in terms of Lemma 2.1, we have

$$
\begin{aligned}
\sum_{j \in J_{K}} a_{j} & \leq K(r) p \leq c_{1} \frac{\mathcal{A}(r)}{\max \left\{\sqrt{\Lambda(r)} \log r, \log ^{\tau} \mathcal{A}(r)\right\}}\left(\frac{r}{\Gamma}+\log r\right) \\
& \leq c_{1}\left(\frac{\mathcal{A}(r)}{\log ^{\eta} \mathcal{A}(r)}+\frac{\mathcal{A}(r)}{\sqrt{\Lambda(r)}}\right) .
\end{aligned}
$$

In terms of Lemma 2.1 again, we have

$$
\sum_{j \in J_{v, \gamma}} a_{j} \leq c_{2} \varepsilon v\left(\Omega\left(r^{\prime}\right)\right) \leq c_{2} \varepsilon\left(\mathcal{A}\left(r^{\prime}\right)+\sum_{k=1}^{q} \mu_{k}\left(\Omega\left(r^{\prime}\right)\right)\right) .
$$

For $j \notin J_{v, \gamma} \cup J_{K}$, we have $a_{j} \geq K=K(r)$. Set $U^{j}(z)=\frac{1}{a_{j}} v\left(\rho_{j} z+z_{j}\right), \quad U_{k}^{j}(z)=\frac{1}{a_{j}} v_{k}\left(\rho_{j} z+z_{j}\right) \quad(1 \leq k \leq q, 1 \leq j \leq p)$ and

$$
\kappa^{j}=\frac{\rho_{j}^{2}}{a_{j}}\left[\sum_{k=1}^{q} \mu_{k}-(q-2 n) \mu\right] .
$$

Then $\kappa^{j}$ is the Riesz charge of $\sum_{k=1}^{q} U_{k}^{j}(z)-(q-2 n) U^{j}$. For any subset $I$ of $\{1,2, \ldots, q\}$ with cardinality $n+1$, we have

$$
\left\|\bigvee_{k \in I} U_{k}^{j}-U^{j}\right\| \leq \frac{1}{a_{j}} \max _{z \in B_{j}}\left|\bigvee_{k \in I} v_{k}^{j}-v^{j}\right| \leq \frac{c}{a_{j}} \leq \frac{c}{K(r)} \rightarrow 0 \quad(r \rightarrow \infty),
$$


where $B_{j}=B\left(z_{j}, \rho_{j}\right)$, and $c$ is a constant only depending on $\mathbf{a}_{1}, \ldots, \mathbf{a}_{q}$. Therefore, by Theorem $2^{\prime}$ of [8] we have

$$
-\varepsilon<\int \psi_{j}(z) \mathrm{d} \kappa^{j}(z),
$$

and by substituting $z$ with $\frac{w-z_{j}}{\rho_{j}}$ we have

$$
\begin{aligned}
-\varepsilon & <\int \varphi_{j}(w)\left|\left(\frac{w-z_{j}}{\rho_{j}}\right)^{\prime}\right|^{2} \mathrm{~d} \frac{\rho_{j}^{2}}{a_{j}}\left[\sum_{k=1}^{q} \mu_{k}-(q-2 n) \mu\right](w) \\
& =\frac{1}{a_{j}}\left[\sum_{k=1}^{q} \int \varphi_{j}(w) \mathrm{d} \mu_{k}(w)-(q-2 n) \int \varphi_{j}(w) \mathrm{d} \mu(w)\right],
\end{aligned}
$$

so that

$$
(q-2 n) \int \varphi_{j} \mathrm{~d} \mu \leq \sum_{k=1}^{q} \int \varphi_{j} \mathrm{~d} \mu_{k}+\varepsilon \int \varphi_{j} \mathrm{~d} \mu .
$$

Combining (2.3), (2.4), and (2.5) yields

$$
\begin{aligned}
(q-2 n) \int \Phi \mathrm{d} \mu \leq & \sum_{k=1}^{q} \int \Phi \mathrm{d} \mu_{k}+\varepsilon \int \Phi \mathrm{d} \mu \\
& +c_{2} \varepsilon\left(\mathcal{A}\left(r^{\prime}\right)+\sum_{k=1}^{q} \mu_{k}\left(\Omega\left(r^{\prime}\right)\right)\right) \\
& +c_{1}\left(\frac{\mathcal{A}(r)}{\log ^{\eta} \mathcal{A}(r)}+\frac{\mathcal{A}(r)}{\sqrt{\Lambda(r)}}\right) .
\end{aligned}
$$

This implies that

$$
\begin{aligned}
(q-2 n) \mathcal{A}\left(r, \Omega_{\epsilon}, f\right) \leq & \sum_{k=1}^{q} n_{\Omega}\left(r^{\prime}, \mathbf{a}_{k}, f\right)+\left(c_{2}+1\right) \varepsilon\left(\mathcal{A}\left(r^{\prime}\right)+\sum_{k=1}^{q} \mu_{k}\left(\Omega\left(r^{\prime}\right)\right)\right) \\
& +c_{1}\left(\frac{\mathcal{A}(r)}{\log ^{\eta} \mathcal{A}(r)}+\frac{\mathcal{A}(r)}{\sqrt{\Lambda(r)}}\right) .
\end{aligned}
$$

The remainder of the proof is the same as the proof of Theorem 1 of [8].

We remark that $\Omega_{\epsilon}$ in Theorem 2.1 cannot be replaced by $\Omega$.

Now we introduce the Nevanlinna characteristic, counting function, and proximity function of holomorphic curves in an angular domain following Nevanlinna (see [12] or [31]) for the case of meromorphic functions. This attempt was made by Fedorov and Grishin [9], who considered a class of more general holomorphic curves, called just holomorphic curves and solved the estimate problem of error terms in the second main theorems by rearranging the counting functions and proximity functions. We will mention those in detail in the sequel.

Let $\Omega$ be the upper half-plane minus the unit disk centered at 0 , and for $r>1$, put $\Omega(r)=\{z: 1<|z|<r, 0<\arg z<\pi\}$. Consider the function 
$u_{r}(z)=-\operatorname{Im}\left(1 / z+z / r^{2}\right)$ on $\Omega(r)$ and the subharmonic function $v(z)$ on $\bar{\Omega}(r)$. Define the Nevanlinna characteristic of $v$ as

$$
\begin{aligned}
S_{\Omega}(r, v) & =\frac{1}{2 \pi} \iint_{\Omega(r)} u_{r}(\zeta) \Delta v(\zeta) \\
& =\frac{1}{2 \pi} \int_{1}^{r} \int_{0}^{\pi}\left(\frac{1}{t}-\frac{t}{r^{2}}\right) \sin \theta \Delta v\left(t e^{i \theta}\right),
\end{aligned}
$$

where we used the equality $u_{r}\left(t e^{i \theta}\right)=\left(1 / t-t / r^{2}\right) \sin \theta$. Sometimes, we also write $S_{0, \pi}(r, v)$ for $S_{\Omega}(r, v)$.

By noting that $\Delta u_{r}(z)=0$ for all $z \neq 0$ and $u_{r}(z)=0$ on $\partial \Omega(r) \backslash \gamma, \gamma=\left\{e^{i \theta}\right.$ : $\theta \in[0, \pi]\}$, in view of the Green formula, we have

$$
\begin{aligned}
S_{\Omega}(r, v) & =\frac{1}{2 \pi} \int_{\partial \Omega(r)} v(\zeta) \frac{\partial u_{r}}{\partial \mathbf{n}} \mathrm{d} s-\frac{1}{2 \pi} \int_{\partial \Omega(r)} u_{r}(\zeta) \frac{\partial v}{\partial \mathbf{n}} \mathrm{d} s \\
& =\frac{1}{2 \pi} \int_{\partial \Omega(r)} v(\zeta) \frac{\partial u_{r}}{\partial \mathbf{n}} \mathrm{d} s-\frac{1}{2 \pi} \int_{\gamma} u_{r}(\zeta) \frac{\partial v}{\partial \mathbf{n}} \mathrm{d} s \\
& =\frac{1}{2 \pi} \int_{\partial \Omega(r)} v(\zeta) \frac{\partial u_{r}}{\partial \mathbf{n}} \mathrm{d} s-\frac{1}{2 \pi} \int_{0}^{\pi}\left(1-\frac{1}{r^{2}}\right) \sin \theta \frac{\partial v}{\partial \mathbf{n}} \mathrm{d} \theta
\end{aligned}
$$

where $\mathbf{n}$ is the inner normal on $\partial \Omega(r)$.

Let $f=\left[f_{0}: f_{1}: \ldots: f_{n}\right]$ be a holomorphic curve on $\bar{\Omega}$, and $\mathbf{f}=\left(f_{0}\right.$, $f_{1}, \ldots, f_{n}$ ) be a reduced representation of $f$. Set

$$
v(z)=\bigvee_{j=0}^{n} \log \left|f_{j}(z)\right|, \quad z \in \Omega .
$$

Define the Nevanlinna characteristic of $f$ as $S_{\Omega}(r, f)=S_{\Omega}(r, v)$, and for a hyperplane $\mathbf{a}=\left(a_{0}, a_{1}, \ldots, a_{n}\right)$, define the counting function $f$ with respect to $\mathbf{a}$ for $\Omega$ as

$$
C_{\Omega}(r, \mathbf{a}, f)=S_{\Omega}\left(r, v_{\mathbf{a}}\right),
$$

where $v_{\mathbf{a}}=\log |\langle\mathbf{f}, a\rangle|$ and $\langle\mathbf{f}, a\rangle=a_{0} f_{0}+a_{1} f_{1}+\cdots+a_{n} f_{n}$. Then

$$
\begin{aligned}
C_{\Omega}(r, \mathbf{a}, f) & =\frac{1}{2 \pi} \int_{1}^{r} \int_{0}^{\pi}\left(\frac{1}{t}-\frac{t}{r^{2}}\right) \sin \theta \Delta v_{\mathbf{a}}\left(t e^{i \theta}\right) \\
& =\sum_{k}\left(\frac{1}{r_{k}}-\frac{r_{k}}{r^{2}}\right) \sin \theta_{k},
\end{aligned}
$$

where $z_{k}=r_{k} e^{i \theta_{k}}$ is a zero of $\langle\mathbf{f}, a\rangle$ on $\Omega(r)$, counted with multiplicities.

Set $\|\mathbf{f}\|=\left(\left|f_{0}\right|^{2}+\left|f_{1}\right|^{2}+\cdots+\left|f_{n}\right|^{2}\right)^{1 / 2}$ and $\|\mathbf{a}\|=\left(\left|a_{0}\right|^{2}+\left|a_{1}\right|^{2}+\cdots+\right.$ $\left.\left|a_{n}\right|^{2}\right)^{1 / 2}$. If $\langle\mathbf{f}, a\rangle \neq 0$, then the Weil function of $f$ with respect to the hyperplane $H$ with a reduced representation $\mathbf{a}$ is

$$
\lambda_{H}(f(z))=\log \frac{\|\mathbf{f}\|\|\mathbf{a}\|}{|\langle\mathbf{f}, a\rangle|} .
$$

Define the proximity functions of $f$ for the hyperplane a on $\Omega$ by

$$
A_{\Omega}(r, \mathbf{a}, f)=\frac{1}{2 \pi} \int_{\Gamma_{r}^{\prime}} \lambda_{H}(f(\zeta)) \frac{\partial u_{r}}{\partial \mathbf{n}} \mathrm{d} s,
$$




$$
B_{\Omega}(r, \mathbf{a}, f)=\frac{1}{2 \pi} \int_{\Gamma_{r}^{\prime \prime}} \lambda_{H}(f(\zeta)) \frac{\partial u_{r}}{\partial \mathbf{n}} \mathrm{d} s,
$$

where $\Gamma_{r}^{\prime \prime}=\left\{r e^{i \theta}: 0 \leq \theta \leq \pi\right\} \cup\left\{e^{i \theta}: 0 \leq \theta \leq \pi\right\}$ and $\Gamma_{r}^{\prime}=\partial \Omega(r) \backslash \Gamma_{r}^{\prime \prime}$. Let

$$
S_{\Omega}(r, \mathbf{a}, f)=A_{\Omega}(r, \mathbf{a}, f)+B_{\Omega}(r, \mathbf{a}, f)+C_{\Omega}(r, \mathbf{a}, f) .
$$

By the Green formula we have

$$
\int_{\partial \Omega(r)} \frac{\partial u_{r}}{\partial \mathbf{n}} \mathrm{d} s=-\iint_{\Omega(r)} \Delta u_{r} \mathrm{~d} \sigma=0 .
$$

Therefore, we have

$$
\begin{aligned}
S_{\Omega}(r, \mathbf{a}, f)= & A_{\Omega}(r, \mathbf{a}, f)+B_{\Omega}(r, \mathbf{a}, f)+C_{\Omega}(r, \mathbf{a}, f) \\
= & \frac{1}{2 \pi} \int_{\partial \Omega(r)} \log \frac{\|\mathbf{f}\|\|\mathbf{a}\|}{|\langle\mathbf{f}, a\rangle|} \frac{\partial u_{r}}{\partial \mathbf{n}} \mathrm{d} s+\frac{1}{2 \pi} \int_{\partial \Omega(r)} v_{\mathbf{a}}(\zeta) \frac{\partial u_{r}}{\partial \mathbf{n}} \mathrm{d} s \\
& -\frac{1}{2 \pi} \int_{0}^{\pi}\left(1-\frac{1}{r^{2}}\right) \sin \theta \frac{\partial v_{\mathbf{a}}}{\partial \mathbf{n}} \mathrm{d} \theta \\
= & \frac{1}{2 \pi} \int_{\partial \Omega(r)} \log \|\mathbf{f}\| \frac{\partial u_{r}}{\partial \mathbf{n}} \mathrm{d} s-\frac{1}{2 \pi} \int_{0}^{\pi}\left(1-\frac{1}{r^{2}}\right) \sin \theta \frac{\partial v_{\mathbf{a}}}{\partial \mathbf{n}} \mathrm{d} \theta .
\end{aligned}
$$

Now we consider the general case $\Omega=\Omega(\alpha, \beta)$ with $0<\beta-\alpha<2 \pi$. The transformation $w=\left(e^{-i \alpha} z\right)^{\omega}$ with $\omega=\frac{\pi}{\beta-\alpha}$ conformally maps $\Omega(\alpha, \beta)$ onto $\Omega(0, \pi)$. Set $F(w)=f\left(e^{i \alpha} w^{1 / \omega}\right)=f(z)$ for a holomorphic curve $f$ on $\bar{\Omega}(\alpha, \beta)$. For a hyperplane a, define

$$
\begin{aligned}
& A_{\alpha, \beta}(r, \mathbf{a}, f)=A_{0, \pi}\left(r^{\omega}, \mathbf{a}, F\right), \\
& B_{\alpha, \beta}(r, \mathbf{a}, f)=B_{0, \pi}\left(r^{\omega}, \mathbf{a}, F\right), \\
& C_{\alpha, \beta}(r, \mathbf{a}, f)=C_{0, \pi}\left(r^{\omega}, \mathbf{a}, F\right), \\
& S_{\alpha, \beta}(r, \mathbf{a}, f)=S_{0, \pi}\left(r^{\omega}, \mathbf{a}, F\right),
\end{aligned}
$$

and also

$$
S_{\alpha, \beta}(r, f)=S_{0, \pi}\left(r^{\omega}, F\right) .
$$

We establish the first main theorem for the characteristics.

THeOREM 2.2. Let $f$ be a holomorphic curve on $\bar{\Omega}(\alpha, \beta)$, and $\mathbf{f}=\left(f_{0}, f_{1}, \ldots, f_{n}\right)$ be a reduced representation of $f$. Then for a hyperplane $\mathbf{a}$, we have

$$
S_{\alpha, \beta}(r, f)=S_{\alpha, \beta}(r, \mathbf{a}, f)+O(1) .
$$

Proof. Clearly, it is sufficient to prove the case where $\Omega=\Omega(0, \pi)$. Set

$$
\dot{S}_{\Omega}(r, f)=\frac{1}{2 \pi} \int_{\partial \Omega(r)} \log \|\mathbf{f}\| \frac{\partial u_{r}}{\partial \mathbf{n}} \mathrm{d} s .
$$

We only need to prove that

$$
S_{\Omega}(r, f)=\dot{S}_{\Omega}(r, f)+O(1) .
$$


Noting the basic inequalities

$$
v(z) \leq \log \|\mathbf{f}\| \leq v(z)+\frac{1}{2} \log (n+1),
$$

$\partial u_{r} / \partial \mathbf{n}>0$ for $z \in \Gamma_{r}=\partial \Omega(r) \backslash \gamma$ and $\partial u_{r} / \partial \mathbf{n}<0$ for $z \in \gamma, \gamma=\left\{e^{i \theta}: \theta \in\right.$ $[0, \pi]\}$, we have

$$
\begin{aligned}
S_{\Omega}(r, f) & =\frac{1}{2 \pi} \int_{\Gamma_{r}} v(\zeta) \frac{\partial u_{r}}{\partial \mathbf{n}} \mathrm{d} s+\frac{1}{2 \pi} \int_{\gamma} v(\zeta) \frac{\partial u_{r}}{\partial \mathbf{n}} \mathrm{d} s \\
& \leq \frac{1}{2 \pi} \int_{\Gamma_{r}} \log \|\mathbf{f}\| \frac{\partial u_{r}}{\partial \mathbf{n}} \mathrm{d} s+\frac{1}{2 \pi} \int_{\gamma} v(\zeta) \frac{\partial u_{r}}{\partial \mathbf{n}} \mathrm{d} s \\
& =\dot{S}_{\Omega}(r, f)-\frac{1}{2 \pi} \int_{\gamma}(\log \|\mathbf{f}\|-v(\zeta)) \frac{\partial u_{r}}{\partial \mathbf{n}} \mathrm{d} s
\end{aligned}
$$

and

$$
\begin{aligned}
\dot{S}_{\Omega}(r, f) & =\frac{1}{2 \pi} \int_{\Gamma_{r}} \log \|\mathbf{f}\| \frac{\partial u_{r}}{\partial \mathbf{n}} \mathrm{d} s+\frac{1}{2 \pi} \int_{\gamma} \log \|\mathbf{f}\| \frac{\partial u_{r}}{\partial \mathbf{n}} \mathrm{d} s \\
& \leq S_{\Omega}(r, f)+\frac{1}{2 \pi} \int_{\gamma}(\log \|\mathbf{f}\|-v(\zeta)) \frac{\partial u_{r}}{\partial \mathbf{n}} \mathrm{d} s+\frac{\log (n+1)}{4 \pi} \int_{\Gamma_{r}} \frac{\partial u_{r}}{\partial \mathbf{n}} \mathrm{d} s \\
& \leq S_{\Omega}(r, f)-\frac{\log (n+1)}{4 \pi} \int_{\gamma} \frac{\partial u_{r}}{\partial \mathbf{n}} \mathrm{d} s \\
& =S_{\Omega}(r, f)+\frac{\log (n+1)}{2 \pi}\left(1+\frac{1}{r^{2}}\right) .
\end{aligned}
$$

Combining these inequalities yields equation (2.7).

Another version of the first main theorem for the characteristics is the following:

Theorem 2.3. Let $\Omega=\Omega(\alpha, \beta)$ with $0<\beta-\alpha<2 \pi$, and $f$ be a holomorphic curve on $\bar{\Omega}$ with a reduced representation $\mathbf{f}$. Let $\mathbf{a}_{0}, \mathbf{a}_{1}, \ldots, \mathbf{a}_{n}$ be $n+1$ hyperplanes in general position. Assume that $g$ is a holomorphic curve determined by

$$
\mathbf{g}(z)=\mathbf{f}(z) A, \quad A=\left(\mathbf{a}_{0}, \mathbf{a}_{1}, \ldots, \mathbf{a}_{n}\right) .
$$

Then

$$
S_{\alpha, \beta}(r, g)=S_{\alpha, \beta}(r, f)+O(1) .
$$

Proof. It is easy to see that there exists a positive constant $C$ such that

$$
C^{-1} \log \|\mathbf{f}\| \leq \log \|\mathbf{g}\| \leq C \log \|\mathbf{f}\| .
$$

Relation (2.9) follows from this inequality and from (2.8).

We say that a holomorphic curve $f$ on $\Omega$ is $m$-nondegenerated representation $\mathbf{f}=\left(f_{0}, \ldots, f_{n}\right)$ if any $m+2$ elements of $f_{0}, \ldots, f_{n}$ are linearly dependent and among them there are $m+1$ linearly independent elements, that is, the linear span of $f_{0}, \ldots, f_{n}$ has dimension $m+1$. If $m=n$, we say that $f$ is nondegenerate. 
In view of the methods of Cartan [1] and Ru and Stoll in [21] (cf. Ru [19] and Gundersen and Hayman [14] and [13]), we establish the second main theorem for a holomorphic curve on an angle.

Theorem 2.4. Let $\Omega=\Omega(\alpha, \beta)$ with $0<\beta-\alpha<2 \pi$, and $f$ be an $m$ nondegenerate holomorphic curve on $\bar{\Omega}(\alpha-\varepsilon, \beta+\varepsilon)$ for some $\varepsilon>0$ with a reduced representation $\mathbf{f}=\left(f_{0}, f_{1}, \ldots, f_{n}\right)$. Let $\mathbf{a}_{1}, \mathbf{a}_{2}, \ldots, \mathbf{a}_{q}$ be $q$ hyperplanes in general position. Then we have

$$
\begin{aligned}
& (q-2 n+m-1) S_{\alpha, \beta}(r, f) \\
& \quad \leq \sum_{k=1}^{q} C_{\alpha, \beta}\left(r, \mathbf{a}_{k}, f\right)-C_{\alpha, \beta}(r, 0, W)+R_{\alpha, \beta}(r, f),
\end{aligned}
$$

where $W$ is the Wronskian of $m+1$ linearly independent elements of $f_{0}$, $f_{1}, \ldots, f_{n}$, and $R_{\alpha, \beta}(r, f)$ is the error term with the estimate

$$
R_{\alpha, \beta}(r, f) \leq K\left(\log ^{+} S_{\alpha-\varepsilon, \beta+\varepsilon}(r, f)+\log ^{+} r+1\right)
$$

for all $r>1$, except possibly a set of finite linear measure, where $K$ is a constant depending on $\varepsilon$.

REMARK 1. If $f$ is a linearly nondegenerate holomorphic curve, then we have

$$
\begin{aligned}
& (q-n-1) S_{\alpha, \beta}(r, f) \\
& \quad \leq \sum_{k=1}^{q} C_{\alpha, \beta}\left(r, \mathbf{a}_{k}, f\right)-C_{\alpha, \beta}(r, 0, W)+R_{\alpha, \beta}(r, f),
\end{aligned}
$$

where $W$ is the Wronskian of $f_{0}, f_{1}, \ldots, f_{n}$.

We can give the truncated second main theorem. By $C_{\alpha, \beta}^{s)}(r, \mathbf{a}, f)$ we denote the counting function in which zero of $\langle\mathbf{f}, a\rangle$ with multiplicity $p$ is counted by $\min \{s, p\}$ times. We have

$$
\begin{aligned}
& \sum_{k=1}^{q} C_{\alpha, \beta}\left(r, \mathbf{a}_{k}, f\right)-C_{\alpha, \beta}(r, 0, W) \\
& \quad \leq \sum_{k=1}^{q} C_{\alpha, \beta}^{m)}\left(r, \mathbf{a}_{k}, f\right)-C_{\alpha, \beta}(r, 0, H),
\end{aligned}
$$

where

$$
H=\frac{h_{1} h_{2} \cdots h_{q}}{W\left(f_{i_{0}}, f_{i_{1}}, \ldots, f_{i_{m}}\right)}, \quad h_{j}=\left\langle\mathbf{f}, a_{j}\right\rangle, \quad 1 \leq j \leq q,
$$

where $\left\{f_{i_{0}}, f_{i_{1}}, \ldots, f_{i_{m}}\right\}$ is a base of linear span of $f_{0}, f_{1}, \ldots, f_{n}$. Note that $W$ is independent of choice of the base.

From (2.12) and (2.13) we can see that Theorem 2.4 is a generalization of the second main theorem for Nevanlinna characteristic of a meromorphic function with ramification term for an angle to a holomorphic curve on an angle.

Now we prepare for the proof of Theorem 2.4. 
Lemma 2.2. Let $f: \Omega \rightarrow \mathcal{P}^{m}(\mathbb{C})$ be a nondegenerate holomorphic curve with a reduced representation $\mathbf{f}=\left(f_{0}, f_{1}, \ldots, f_{m}\right)$. Let $\mathbf{a}_{1}, \mathbf{a}_{2}, \ldots, \mathbf{a}_{q}$ be $q$ hyperplanes on $\mathcal{P}^{m}(\mathbb{C})$.

(I) (Cartan [1]) Assume that $\mathbf{a}_{1}, \mathbf{a}_{2}, \ldots, \mathbf{a}_{q}$ are in $s$-general position and $\omega(j) \geq 0(1 \leq j \leq q)$. Then there exists a positive constant $C$ such that for all $z \in \Omega$,

$$
\prod_{j=1}^{q}\left(\frac{\|\mathbf{f}(z)\|\left\|\mathbf{a}_{j}\right\|}{\left|\left\langle\mathbf{f}(z), \mathbf{a}_{j}\right\rangle\right|}\right)^{\omega(j)} \leq C \max _{1 \leq i_{0}<i_{1}<\cdots<i_{s} \leq q} \prod_{k=0}^{s}\left(\frac{\|\mathbf{f}(z)\|\left\|\mathbf{a}_{i_{k}}\right\|}{\left|\left\langle\mathbf{f}(z), \mathbf{a}_{i_{k}}\right\rangle\right|}\right)^{\omega\left(i_{k}\right)},
$$

where max is taken over all $\mathbf{a}_{i_{k}}(k=0,1, \ldots, s)$;

(II) (Nochka, see Thm. A3.4.3 in [19]) Assume that $\mathbf{a}_{1}, \mathbf{a}_{2}, \ldots, \mathbf{a}_{q}$ are in $n$ subgeneral position. Then there exists a positive constant $C$ such that for all $z \in \Omega$ and for any $M \subset\{1,2, \ldots, q\}$ with $\# M \leq n+1$, we have

$$
\prod_{j \in M}\left(\frac{\|\mathbf{f}(z)\|\left\|\mathbf{a}_{j}\right\|}{\left|\left\langle\mathbf{f}(z), \mathbf{a}_{j}\right\rangle\right|}\right)^{\omega(j)} \leq C \max _{1 \leq i_{0}<i_{1}<\cdots<i_{m} \leq q} \prod_{k=0}^{m} \frac{\|\mathbf{f}(z)\|\left\|\mathbf{a}_{i_{k}}\right\|}{\left|\left\langle\mathbf{f}(z), \mathbf{a}_{i_{k}}\right\rangle\right|},
$$

where max is taken over all linearly independent group $\mathbf{a}_{i_{k}}(k=0,1, \ldots, m)$, and $\omega(j)$ is the Nochka weight for $\mathbf{a}_{j}$ with properties that for a real number $\frac{n+1}{m+1} \leq \theta \leq \frac{2 n-m+1}{m+1}$, we have

$$
0 \leq \omega(j) \theta \leq 1, \quad q-2 n+m-1=\theta\left(\sum_{j=1}^{q} \omega(j)-m-1\right) .
$$

Proof. To prove (I), for any $z \in \Omega$, we can write

$$
\left|\left\langle\mathbf{f}(z), \mathbf{a}_{i_{0}}\right\rangle\right| \leq\left|\left\langle\mathbf{f}(z), \mathbf{a}_{i_{1}}\right\rangle\right| \leq \cdots \leq\left|\left\langle\mathbf{f}(z), \mathbf{a}_{i_{s}}\right\rangle\right| \leq\left|\left\langle\mathbf{f}(z), \mathbf{a}_{j}\right\rangle\right|,
$$

$j \neq i_{k}(k=0,1, \ldots, s) ;\left\langle\mathbf{f}(z), \mathbf{a}_{i_{0}}\right\rangle,\left\langle\mathbf{f}(z), \mathbf{a}_{i_{1}}\right\rangle, \ldots,\left\langle\mathbf{f}(z), \mathbf{a}_{i_{s}}\right\rangle$ are linearly independent, and every $f_{j}$ can be linearly expressed by these $s+1$ functions, and then there exists a positive constant $C_{i_{0}, i_{1}, \ldots, i_{s}}$ such that

$$
\|\mathbf{f}(z)\|\left\|\mathbf{a}_{j}\right\| \leq C_{i_{0}, i_{1}, \ldots, i_{s}}\left|\left\langle\mathbf{f}(z), \mathbf{a}_{j}\right\rangle\right|
$$

$j \neq i_{k}(k=0,1, \ldots, s)$. This yields

$$
\prod_{j=1}^{q}\left(\frac{\|\mathbf{f}(z)\|\left\|\mathbf{a}_{j}\right\|}{\left|\left\langle\mathbf{f}(z), \mathbf{a}_{j}\right\rangle\right|}\right)^{\omega(j)} \leq C_{i_{0}, i_{1}, \ldots, i_{s}}^{\sum_{j \neq i_{k}} \omega(j)} \prod_{k=0}^{s}\left(\frac{\|\mathbf{f}(z)\|\left\|\mathbf{a}_{i_{k}}\right\|}{\left|\left\langle\mathbf{f}(z), \mathbf{a}_{i_{k}}\right\rangle\right|}\right)^{\omega\left(i_{k}\right)} .
$$

Inequality (2.14) follows with $C=\max \left\{C_{i_{0}, i_{1}, \ldots, i_{s}}^{\sum_{j \neq i_{k}} \omega(j)}: 1 \leq i_{0}<i_{1}<\cdots<i_{s} \leq q\right\}$. Result (II) follows immediately from Nochka's result (see Thm. A3.4.3 in [19]). 
The following is Theorem 2.5.1 of [31].

Lemma 2.3. Let $g(z)$ be a meromorphic function on $\Omega(\alpha-\varepsilon, \beta+\varepsilon)$ for $\varepsilon>0$ and $0<\beta-\alpha<2 \pi$. Then

$$
(A+B)_{\alpha, \beta}\left(r, \frac{g^{(k)}}{g}\right) \leq K\left(\log ^{+} S_{\alpha-\varepsilon, \beta+\varepsilon}(r, g)+\log ^{+} r+1\right)
$$

for all $r>1$, except possibly a set of finite linear measure, where $K$ is a constant depending on $\varepsilon$.

Generally, we cannot estimate $A_{\alpha, \beta}\left(r, g^{\prime} / g\right)$ in terms of $\log ^{+} S_{\alpha, \beta}(r, g)$ and $\log ^{+} r$. See Goldberg [11] for a detailed example. If $g$ is meromorphic on the complex plane, then a nice estimate in terms of $\log T(r, g)$ was given by Goldberg and Ostrovskii [12]:

$$
A_{\alpha, \beta}\left(r, \frac{g^{\prime}}{g}\right) \leq K\left[\left(\frac{R}{r}\right)^{\omega} \int_{1}^{R} \frac{\log T(t, g)}{t^{1+\omega}} \mathrm{d} t+\log \frac{r}{R-r}+\log \frac{R}{r}\right]
$$

and

$$
B_{\alpha, \beta}\left(r, \frac{g^{\prime}}{g}\right) \leq \frac{4 \omega}{r^{\omega}} m\left(r, \frac{g^{\prime}}{g}\right) .
$$

An important result is that if $\int^{\infty} t^{-1-\omega} \log ^{+} T(t, g) \mathrm{d} t<\infty$ with $\omega=\frac{\pi}{\beta-\alpha}$, then $(A+B)_{\alpha, \beta}\left(r, g^{\prime} / g\right)=O(1)(r \rightarrow \infty)$.

Lemma 2.4. Let $h_{0}, h_{1}, \ldots, h_{n}$ be $n+1$ analytic functions on $\bar{\Omega}(\alpha-\varepsilon, \beta+\varepsilon)$. Set

$$
\hat{W}(z)=\frac{W\left(h_{0}, h_{1}, \ldots, h_{n}\right)}{h_{0} h_{1} \cdots h_{n}}
$$

and $h=\left[h_{0}: h_{1}: \ldots: h_{n}\right]$. Then

$$
(A+B)_{\alpha, \beta}(r, \hat{W}) \leq K\left(\log ^{+} S_{\alpha-\varepsilon, \beta+\varepsilon}(r, h)+\log ^{+} r+1\right),
$$

for all $r>1$, except possibly a set of finite linear measure, where $K$ is a constant depending on $\varepsilon$.

Proof. Set

$$
g_{j}=\frac{h_{j}}{h_{0}}, \quad j=1,2, \ldots, n .
$$

By the Leibniz formula we have

$$
\left(h_{j}\right)^{(k)}=\left(h_{0} g_{j}\right)^{(k)}=\sum_{i=0}^{k} C_{k}^{i}\left(h_{0}\right)^{(i)}\left(g_{j}\right)^{(k-i)},
$$

so that a simple calculation yields

$$
\begin{aligned}
W\left(h_{0}, h_{1}, \ldots, h_{n}\right) & =W\left(1, g_{1}, g_{2}, \ldots, g_{n}\right) h_{0}^{n+1} \\
& =W\left(g_{1}^{\prime}, g_{2}^{\prime}, \ldots, g_{n}^{\prime}\right) h_{0}^{n+1} .
\end{aligned}
$$


Thus,

$$
\begin{aligned}
|\hat{W}(z)| & =\left|\frac{W\left(g_{1}^{\prime}, g_{2}^{\prime}, \ldots, g_{n}^{\prime}\right)}{g_{1} g_{2} \cdots g_{n}}\right| \\
& \leq \sum_{1 \leq i_{1}+i_{2}+\cdots+i_{n} \leq n(n+1) / 2}\left|\frac{g_{1}^{\left(i_{1}\right)}}{g_{1}} \cdots \frac{g_{n}^{\left(i_{n}\right)}}{g_{n}}\right| .
\end{aligned}
$$

Set $V=\Omega(\alpha-\varepsilon, \beta+\varepsilon)$ and $\hat{u}_{r}(z)=u_{r}\left(e^{i(\alpha-\varepsilon)} z^{1 / \hat{\omega}}\right), \hat{\omega}=\frac{\pi}{\beta-\alpha+2 \varepsilon}$. In view of Lemma 2.3, we have

$$
\begin{aligned}
(A+B)_{\alpha, \beta}(r, \hat{W}) & =\frac{1}{2 \pi} \int_{\partial \Omega(r)} \log ^{+}|\hat{W}| \frac{\partial u_{r}}{\partial \mathbf{n}} \mathrm{d} s \\
& \leq \sum_{j=1}^{n} \sum_{k=1}^{n} \frac{1}{2 \pi} \int_{\partial \Omega(r)} \log ^{+}\left|\frac{g_{j}^{(k)}}{g_{j}}\right| \frac{\partial u_{r}}{\partial \mathbf{n}} \mathrm{d} s+O(1) \\
& =\sum_{j=1}^{n} \sum_{k=1}^{n}(A+B)_{\alpha, \beta}\left(r, \frac{g_{j}^{(k)}}{g_{j}}\right)+O(1) \\
& \leq K\left(\sum_{j=1}^{n} \log ^{+} S_{V}\left(r, g_{j}\right)+\log ^{+} r+1\right) .
\end{aligned}
$$

We estimate

$$
\begin{aligned}
S_{V}\left(r, g_{j}\right) & =\frac{1}{2 \pi} \int_{\partial V(r)}\left(\log \left|h_{0}\right| \vee \log \left|h_{j}\right|\right) \frac{\partial \hat{u}_{r}}{\partial \mathbf{n}} \mathrm{d} s \\
& \leq \frac{1}{2 \pi} \int_{\partial V(r)} \bigvee_{j=0}^{n} \log \left|h_{j}\right| \frac{\partial \hat{u}_{r}}{\partial \mathbf{n}} \mathrm{d} s+O(1) \\
& =S_{V}(r, h)+O(1) .
\end{aligned}
$$

Then we can obtain the desired inequality.

From the proof of Lemma 2.4 it is easy to see that estimates of $A_{\alpha, \beta}(r, \hat{W})$ and $B_{\alpha, \beta}(r, \hat{W})$ analogous to those of Goldberg and Ostrovskii [12] can be also established in view of $\log T(r, h)$ when $h$ is a holomorphic curve on the complex plane.

Now we are in the position to prove Theorem 2.4.

Proof of Theorem 2.4. It is sufficient to prove our result for the case where $\Omega=$ $\Omega(0, \pi)$. Assume that $f_{0}, f_{1}, \ldots, f_{m}$ are linearly independent and

$$
f_{j}=c_{0}^{(j)} f_{0}+c_{1}^{(j)} f_{1}+\cdots+c_{m}^{(j)} f_{m}, \quad m+1 \leq j \leq n .
$$


Write $\mathbf{g}=\left(f_{0}, f_{1}, \ldots, f_{m}\right), \mathbf{a}_{j}=\left(a_{0}^{(j)}, a_{1}^{(j)}, \ldots, a_{n}^{(j)}\right)$, and $\mathbf{b}_{j}=\left(b_{0}^{(j)}, b_{1}^{(j)}, \ldots\right.$, $\left.b_{m}^{(j)}\right), 1 \leq j \leq q$, where $b_{t}^{(j)}=a_{t}^{(j)}+a_{m+1}^{(j)} c_{t}^{(m+1)}+\cdots+a_{n}^{(j)} c_{t}^{(n)}, 0 \leq t \leq m$. Then $g=\left[f_{0}: f_{1}: \ldots: f_{m}\right]: \Omega \rightarrow \mathcal{P}^{m}(\mathbb{C})$ is a linearly nondegenerate holomorphic curve, and

$$
S_{\Omega}(r, f)=S_{\Omega}(r, g)+O(1) .
$$

Since $\left\langle\mathbf{f}, a_{j}\right\rangle=\left\langle\mathbf{g}, b_{j}\right\rangle, 1 \leq j \leq q$, we have

$$
C_{\Omega}\left(r, \mathbf{a}_{j}, f\right)=C_{\Omega}\left(r, \mathbf{b}_{j}, g\right)
$$

and the Weil functions $\lambda_{\mathbf{a}_{j}}(f(z))=\lambda_{\mathbf{b}_{j}}(g(z))+O(1)$, and further

$$
\begin{aligned}
& A_{\Omega}\left(r, \mathbf{a}_{j}, f\right)=A_{\Omega}\left(r, \mathbf{b}_{j}, g\right)+O(1), \\
& B_{\Omega}\left(r, \mathbf{a}_{j}, f\right)=B_{\Omega}\left(r, \mathbf{b}_{j}, g\right)+O(1),
\end{aligned}
$$

$1 \leq j \leq q$.

Since $\mathbf{a}_{1}, \mathbf{a}_{2}, \ldots, \mathbf{a}_{q}$ are in general position, $\mathbf{b}_{1}, \mathbf{b}_{2}, \ldots, \mathbf{b}_{q}$ are in $n$-subgeneral position. In terms of result (II) in Lemma 2.2, for any $M \subset\{1,2, \ldots, q\}$ with $\# M=n+1$, we have

$$
\begin{aligned}
& \sum_{j \in M} \omega(j) \frac{1}{2 \pi} \int_{\partial \Omega(r)} \lambda_{\mathbf{b}_{j}}(g(z)) \frac{\partial u_{r}}{\partial \mathbf{n}} \mathrm{d} s \\
& \leq \frac{1}{2 \pi} \int_{\partial \Omega(r)} \log \|g\|^{m+1} \frac{\partial u_{r}}{\partial \mathbf{n}} \mathrm{d} s \\
&+\max _{1 \leq i_{0}<i_{1}<\cdots<i_{m} \leq q} \frac{1}{2 \pi} \int_{\partial \Omega(r)} \log \prod_{k=0}^{m} \frac{1}{\left|w_{\mathbf{b}_{i_{k}}}\right|} \frac{\partial u_{r}}{\partial \mathbf{n}} \mathrm{d} s+O(1) \\
&=(m+1) S_{\Omega}(r, g)+\max _{1 \leq i_{0}<i_{1}<\cdots<i_{m} \leq q} \frac{1}{2 \pi} \int_{\partial \Omega(r)} \log \left|\frac{W}{\prod_{k=0}^{m} w_{\mathbf{b}_{i_{k}}}}\right| \frac{\partial u_{r}}{\partial \mathbf{n}} \mathrm{d} s \\
&-\frac{1}{2 \pi} \int_{\partial \Omega(r)} \log |W| \frac{\partial u_{r}}{\partial \mathbf{n}} \mathrm{d} s+O(1) \\
&=(m+1) S_{\Omega}(r, f)-C_{\Omega}(r, 0, W)+R_{\Omega}(r, g),
\end{aligned}
$$

where $w_{\mathbf{b}_{i_{k}}}=\left\langle\mathbf{g}, \mathbf{b}_{i_{k}}\right\rangle$ and $W=W\left(f_{0}, f_{1}, \ldots, f_{m}\right)$.

In terms of result (I) in Lemma 2.2, we have

$$
\begin{aligned}
& \sum_{j=1}^{q} \omega(j) \frac{1}{2 \pi} \int_{\partial \Omega(r)} \lambda_{\mathbf{a}_{j}}(f(z)) \frac{\partial u_{r}}{\partial \mathbf{n}} \mathrm{d} s \\
& \quad \leq \max _{M} \sum_{j \in M} \omega(j) \frac{1}{2 \pi} \int_{\partial \Omega(r)} \lambda_{\mathbf{a}_{j}}(f(z)) \frac{\partial u_{r}}{\partial \mathbf{n}} \mathrm{d} s+O(1),
\end{aligned}
$$

where max is taken over all $M \subset\{1,2, \ldots, q\}$ with $\# M=n+1$. Thus, combining the last two inequalities yields that

$$
\sum_{j=1}^{q} \omega(j)\left(A_{\Omega}+B_{\Omega}\right)\left(r, \mathbf{a}_{j}, f\right) \leq(m+1) S_{\Omega}(r, f)-C_{\Omega}(r, 0, W)+R_{\Omega}(r, g),
$$


so that

$$
\begin{aligned}
& \frac{1}{2 \pi} \int_{\partial \Omega(r)} \log \frac{\|f\|^{q}}{\prod_{j=1}^{q}\left|\left\langle\mathbf{f}, a_{j}\right\rangle\right|} \frac{\partial u_{r}}{\partial \mathbf{n}} \mathrm{d} s \\
&=\sum_{j=1}^{q}\left(A_{\Omega}+B_{\Omega}\right)\left(r, \mathbf{a}_{j}, f\right)+O(1) \\
&=\sum_{j=1}^{q}(1-\theta \omega(j))\left(A_{\Omega}+B_{\Omega}\right)\left(r, \mathbf{a}_{j}, f\right) \\
& \quad+\theta \sum_{j=1}^{q} \omega(j)\left(A_{\Omega}+B_{\Omega}\right)\left(r, \mathbf{a}_{j}, f\right)+O(1) \\
& \leq \sum_{j=1}^{q}(1-\theta \omega(j)) S_{\Omega}(r, f)+\theta(m+1) S_{\Omega}(r, f) \\
& \quad-\theta C_{\Omega}(r, 0, W)+\theta R_{\Omega}(r, g) \\
& \leq(2 n-m+1) S_{\Omega}(r, f)-\frac{n+1}{m+1} C_{\Omega}(r, 0, W)+R_{\Omega}(r, g) .
\end{aligned}
$$

In view of the definition of $S_{\Omega}(r, f)$ and Theorem 2.2, we have

$$
\begin{aligned}
q S_{\Omega}(r, f)= & \frac{1}{2 \pi} \int_{\partial \Omega(r)} \log \|f\|^{q} \frac{\partial u_{r}}{\partial \mathbf{n}} \mathrm{d} s+O(1) \\
= & \sum_{k=1}^{q} C_{\Omega}\left(r, \mathbf{a}_{k}, f\right)+\frac{1}{2 \pi} \int_{\partial \Omega(r)} \log \frac{\|f\|^{q}}{\prod_{j=1}^{q}\left|\left\langle\mathbf{f}, a_{j}\right\rangle\right|} \frac{\partial u_{r}}{\partial \mathbf{n}} \mathrm{d} s+O(1) \\
\leq & \sum_{k=1}^{q} C_{\Omega}\left(r, \mathbf{a}_{k}, f\right)+(2 n-m+1) S_{\Omega}(r, f) \\
& -\frac{n+1}{m+1} C_{\Omega}(r, 0, W)+R_{\Omega}(r, g),
\end{aligned}
$$

where

$$
R_{\Omega}(r, g)=(A+B)_{\Omega}(r, \hat{W})+O(1)
$$

with

$$
\hat{W}=\frac{W\left(w_{\mathbf{b}_{i_{0}}}, w_{\mathbf{b}_{i_{1}}}, \ldots, w_{\mathbf{b}_{i_{m}}}\right)}{\left|w_{\mathbf{b}_{i_{0}}} w_{\mathbf{b}_{i_{1}}} \cdots w_{\mathbf{b}_{i_{m}}}\right|} .
$$

Applying Lemma 2.4 to this equality yields the desired result.

The estimate of error terms in Theorem 2.4 is unsatisfactory. In order to overcome that, Fedorov and Grishin [9] modified the definitions of counting functions and proximity functions. The important method in [9] is that a complete measure of a so-called just subharmonic function on the upper half-plane is introduced. The complete measure plays a similar role to the Riesz measure in the previous discussion. In view of the complete measure, they gave definitions of the characteristic, 
counting function, and proximity function and established the following result by virtue of the potential theory of Eremenko and Sodin [8].

THEOREM A. Suppose the assumptions of Theorem 2.1 are satisfied. Then

$$
(q-2 n) A(r, f) \leq \sum_{k=1}^{q} n\left(r, \mathbf{a}_{k}, f\right)+o(A(r, f))+o\left(r \log ^{\sigma} r\right), \quad r \notin E,
$$

where $A(r, f)=v_{v}(B(0, r))$ and $n\left(r, \mathbf{a}_{k}, f\right)=v_{v_{\mathbf{a}_{k}}}(B(0, r))$ for the complete measure $v_{v}$ of $v(z)$ and the complete measure $v_{\mathbf{a}_{k}}$ of $v_{\mathbf{a}_{k}}=\log \left|\left\langle\mathbf{f}, a_{k}\right\rangle\right|$, and

$$
(q-2 n) T(r, f) \leq \sum_{k=1}^{q} N\left(r, \mathbf{a}_{k}, f\right)+o(T(r, f)), \quad r \notin E,
$$

where

$$
T(r, f)=\int_{r_{0}}^{r} \frac{A(t, f)}{t^{3}} \mathrm{~d} t \quad \text { and } \quad N\left(r, \mathbf{a}_{k}, f\right)=\int_{r_{0}}^{r} \frac{n\left(t, \mathbf{a}_{k}, f\right)}{t^{3}} \mathrm{~d} t .
$$

A calculation yields that $N(r, \mathbf{a}, f)=\frac{\pi}{2}\left(A_{\Omega}(r, \mathbf{a}, f)+C_{\Omega}(r, \mathbf{a}, f)+O(1)\right)$ and $m(r, \mathbf{a}, f)=\frac{\pi}{2} B_{\Omega}(r, \mathbf{a}, f)+O(1)$ and the characteristic $T(r, f)=\frac{\pi}{2} S_{\Omega}(r, f)+$ $O(1)$ for the upper half plane $\Omega$. Obviously, we cannot obtain Theorem 2.4 from Theorem A.

Now we introduce the Tsuji characteristic of a holomorphic curve in an angle. Consider the domain $\Xi(r)=\{z:|z-i r / 2|<r / 2\} \backslash\{z:|z|<1\}$, the boundary $\Upsilon_{r}$ of $\Xi(r)$, and $\hat{u}_{r}(z)=-\operatorname{Im}\left(\frac{1}{z}+\frac{i}{r}\right)$. Define

$$
\begin{aligned}
\mathfrak{T}_{\Xi}(r, f) & =\frac{1}{2 \pi} \iint_{\Xi(r)} \hat{u}_{r}(\zeta) \Delta v(\zeta) \\
& =\frac{1}{2 \pi} \int_{0}^{\pi} \int_{1}^{r \sin \theta}\left(\frac{\sin \theta}{t}-\frac{1}{r}\right) \Delta v\left(t e^{i \theta}\right) .
\end{aligned}
$$

By the Green formula and noting $\hat{u}_{r}(z)=0$ on $\{z:|z-i r / 2|=r / 2\}$, we have

$$
\mathfrak{T}_{\Xi}(r, f)=\frac{1}{2 \pi} \int_{\partial \Xi(r)} v(\zeta) \frac{\partial \hat{u}_{r}}{\partial \mathbf{n}} \mathrm{d} s-\frac{1}{2 \pi} \int_{\arcsin r^{-1}}^{\pi-\arcsin r^{-1}}\left(\sin \theta-\frac{1}{r}\right) \frac{\partial v}{\partial \mathbf{n}} \mathrm{d} \theta .
$$

The proximity function $\mathfrak{M}_{\Xi}(r, \mathbf{a}, f)$, counting function $\mathfrak{N}_{\Xi}(r, \mathbf{a}, f)$, and characteristic $\mathfrak{T}_{\Xi}(r, \mathbf{a}, f)$ in the sense of Tsuji are defined as follows:

$$
\begin{aligned}
\mathfrak{N}_{\Xi}(r, \mathbf{a}, f) & =\frac{1}{2 \pi} \int_{\partial \Xi(r)} \lambda_{H}(f(\zeta)) \frac{\partial \hat{u}_{r}}{\partial \mathbf{n}} \mathrm{d} s, \\
\mathfrak{N}_{\Xi}(r, \mathbf{a}, f) & =\frac{1}{2 \pi} \int_{\partial \Xi(r)} v_{\mathbf{a}}(\zeta) \frac{\partial \hat{u}_{r}}{\partial \mathbf{n}} \mathrm{d} s-\frac{1}{2 \pi} \int_{\arcsin r^{-1}}^{\pi-\arcsin r^{-1}}\left(\sin \theta-\frac{1}{r}\right) \frac{\partial v_{\mathbf{a}}}{\partial \mathbf{n}} \mathrm{d} \theta,
\end{aligned}
$$

and

$$
\mathfrak{T}_{\Xi}(r, \mathbf{a}, f)=\mathfrak{M}_{\Xi}(r, \mathbf{a}, f)+\mathfrak{N}_{\Xi}(r, \mathbf{a}, f),
$$


where $v_{\mathbf{a}}=\log |\langle\mathbf{f}, a\rangle|$. Noting that $\hat{u}_{r}$ is harmonic on $\Xi(r)$, we have

$$
\mathfrak{T}_{\Xi}(r, \mathbf{a}, f)=\frac{1}{2 \pi} \int_{\partial \Xi(r)} \log \|\mathbf{f}\| \frac{\partial \hat{u}_{r}}{\partial \mathbf{n}} \mathrm{d} s-\frac{1}{2 \pi} \int_{\arcsin r^{-1}}^{\pi-\arcsin r^{-1}}\left(\sin \theta-\frac{1}{r}\right) \frac{\partial v_{\mathbf{a}}}{\partial \mathbf{n}} \mathrm{d} \theta
$$

and

$$
\begin{aligned}
\mathfrak{N}_{\Xi}(r, \mathbf{a}, f) & =\frac{1}{2 \pi} \int_{0}^{\pi} \int_{1}^{r \sin \theta}\left(\frac{\sin \theta}{t}-\frac{1}{r}\right) \Delta v_{\mathbf{a}}\left(t e^{i \theta}\right) \\
& =\sum_{k}\left(\frac{\sin \theta_{k}}{r_{k}}-\frac{1}{r}\right),
\end{aligned}
$$

where $z_{k}=r_{k} e^{i \theta_{k}}$ is a zero of $\langle\mathbf{f}, a\rangle$ on $\Xi(r)$, counted with multiplicities.

Now consider the general domain

$$
\Xi(\alpha, \beta ; r)=\left\{z=t e^{i \theta}: \alpha<\theta<\beta, 1<t<r(\sin (\omega(\theta-\alpha)))^{1 / \omega}\right\},
$$

$0<\beta-\alpha \leq 2 \pi$ and $\omega=\frac{\pi}{\beta-\alpha}$. The transformation $w=e^{i \alpha} z^{1 / \omega}$ maps conformally $\Xi\left(0, \pi ; r^{\omega}\right)$ onto $\Xi(\alpha, \beta ; r)$. Set $F(z)=f\left(e^{i \alpha} z^{1 / \omega}\right)=f(w)$. Define

$$
\begin{aligned}
\mathfrak{M}_{\alpha, \beta}(r, \mathbf{a}, f) & =\mathfrak{M}_{0, \pi}\left(r^{\omega}, \mathbf{a}, F\right), \\
\mathfrak{N}_{\alpha, \beta}(r, \mathbf{a}, f) & =\mathfrak{N}_{0, \pi}\left(r^{\omega}, \mathbf{a}, F\right), \\
\mathfrak{T}_{\alpha, \beta}(r, \mathbf{a}, f) & =\mathfrak{T}_{0, \pi}\left(r^{\omega}, \mathbf{a}, F\right)
\end{aligned}
$$

for a hyperplane a, and

$$
\mathfrak{T}_{\alpha, \beta}(r, f)=\mathfrak{T}_{0, \pi}\left(r^{\omega}, F\right) .
$$

We have the first main theorems for the Tsuji characteristics, that is, Theorem 2.2 and Theorem 2.3 hold for $\mathfrak{T}_{\Xi}$ instead of $S_{\Omega}$. However, we have a precise second main theorem for the Tsuji characteristics.

THEOREM 2.5. Let $\Omega=\{z: \alpha<\arg z<\beta\}$, and $f$ be a linearly $m$-nondegenerate holomorphic curve on $\Omega$. Let $\mathbf{a}_{1}, \mathbf{a}_{2}, \ldots, \mathbf{a}_{q}$ be $q$ hyperplanes in general position. Then we have

$(q-2 n+m-1) \mathfrak{T}_{\alpha, \beta}(r, f) \leq \sum_{k=1}^{q} \mathfrak{N}_{\alpha, \beta}\left(r, \mathbf{a}_{k}, f\right)-\mathfrak{N}_{\alpha, \beta}(r, 0, W)+Q_{\alpha, \beta}(r, f)$,

where $W$ is the Wronskian of $m+1$ linearly independent elements of $f_{0}$, $f_{1}, \ldots, f_{n}$, and $Q_{\alpha, \beta}(r, f)$ is called the error term with the estimate

$$
Q_{\alpha, \beta}(r, f) \leq K\left(\log ^{+} \mathfrak{T}_{\alpha, \beta}(r, f)+\log ^{+} r+1\right),
$$

for all $r>1$, except possibly a set of finite linear measure, and $K$ is a constant.

We remark on the estimate of the error term $Q_{\alpha, \beta}(r, f)$ in Theorem 2.5. Generally, as we pointed out, $\Omega(\alpha-\varepsilon, \beta+\varepsilon)$ in (2.11) in Theorem 2.4 and $\Omega_{\epsilon}$ in Theorem 2.1 cannot be replaced by $\Omega$. However, if we use the Tsuji domain $\Xi(\alpha, \beta)$, then (2.1) and (2.2) hold with $\Xi(\alpha, \beta)$ instead of $\Omega_{\epsilon}$ and $\Omega$. 


\section{Singular Directions of Holomorphic Curves}

Picard's theorem says that a transcendental meromorphic function on the complex plane $\mathbb{C}$ takes infinitely often all but at most two values on the extended complex plane $\hat{\mathbb{C}}$. An extension of Picard's theorem is Borel's theorem, which describes the number of points at which a transcendental meromorphic function takes some fixed values in terms of the growth order of the function in question, that is, there exist at most two values that have the convergent exponents less than the growth order of the function. The second main theorem of Nevanlinna refines the Borel theorem, and it implies that

$$
\limsup _{r \rightarrow \infty} \frac{N(r, \mathbb{C}, f=a)}{T(r, f)}>0
$$

for all but at most two values of $a$ on $\hat{\mathbb{C}}$. These results stem from the fact that $\infty$ is an essential singular point of a transcendental meromorphic function and essentially reveal the singular phenomenon in the neighborhood of an essential singular point, which does not happen at other points.

The possibility of the same singular phenomenon along a direction is also of interest. A ray is singular for a transcendental meromorphic function if the function takes abundantly all values with exception of at most two values in any angle containing the ray. The word "abundantly" is expressed by "infinitely often" for the Julia direction, whose existence was proved by G. Julia in 1920 for all entire function and by Milloux in 1924 and Valiron in 1938 for most of meromorphic functions; The word "abundantly" is expressed by the growth order of the function in question for the Borel directions, whose existence was proved by G. Valiron in 1938 for a meromorphic function of positive order; We made an expression of it by comparing the integrated counting function in an angle to the Nevanlinna characteristic so that we introduce a new singular direction, called $T$-direction. The second main theorem of Nevanlinna is considered as its background.

In this section, as an application of the Ahlfors-Shimizu second main theorem (Theorem 2.1) for a holomorphic curve in an angle, we discuss the singular directions of a holomorphic curve on $\mathbb{C}$. Let $f$ be a holomorphic curve on the complex plane $\mathbb{C}$ with a reduced representation $\mathbf{f}$. Then the Cartan characteristic of $f$ is

$$
T(r, f)=\frac{1}{2 \pi} \int_{0}^{2 \pi} v\left(r e^{i \theta}\right) \mathrm{d} \theta-v(0) .
$$

Then $T(r, f)=\mathcal{T}(r, \mathbb{C}, f)$. In the sequel, we simply write $\mathcal{A}(r, f)$ and $\mathcal{T}(r, f)$ for $\mathcal{A}(r, \mathbb{C}, f)$ and $\mathcal{T}(r, \mathbb{C}, f)$. The order and lower order of a holomorphic curve $f$ on the complex plane are respectively defined by

$$
\lambda(f)=\limsup _{r \rightarrow \infty} \frac{\log T(r, f)}{\log r}, \quad \tau(f)=\liminf _{r \rightarrow \infty} \frac{\log T(r, f)}{\log r} .
$$


Definition 3.1. A ray $\arg z=\theta$ is called a $T$-direction of a holomorphic curve $f$ if for arbitrary $\varepsilon>0$, we have

$$
\limsup _{r \rightarrow \infty} \frac{N_{Z_{\varepsilon}(\theta)}(r, \mathbf{a}, f)}{T(r, f)}>0,
$$

where $Z_{\varepsilon}(\theta)=\{z: \theta-\varepsilon<\arg z<\theta+\varepsilon\}$, for all hyperplanes a, except possibly those in the linear spaces generated by $m(\leq n)$ elements of at most $2 n$ fixed hyperplanes in general position (this will simply be said to be with exception of at most $2 n$ hyperplanes).

A ray $\arg z=\theta$ is called a Borel direction of a holomorphic curve $f$ of order $\rho$ if for arbitrary $\varepsilon>0,(3.1)$ is replaced with

$$
\limsup _{r \rightarrow \infty} \frac{\log N_{Z_{\varepsilon}(\theta)}(r, \mathbf{a}, f)}{\log r} \geq \rho .
$$

A ray $\arg z=\theta$ is called a Julia direction if for arbitrary $\varepsilon>0,(3.1)$ is replaced with

$$
\lim _{r \rightarrow \infty} N_{Z_{\varepsilon}(\theta)}(r, \mathbf{a}, f)=\infty
$$

REMARK 2. We remark on the exceptional hyperplanes for (3.1) in Definition 3.1: there exist $p(\leq 2 n)$ hyperplanes $\left\{\mathbf{a}_{1}, \ldots, \mathbf{a}_{p}\right\}$ in general position such that all exceptional hyperplanes are in the linear spaces generated by $m(\leq n)$ hyperplanes $\left\{\mathbf{a}_{i_{1}}, \ldots, \mathbf{a}_{i_{m}}\right\}\left(i_{1}<i_{2}<\cdots<i_{m}\right)$. Therefore, for a nonexceptional hyperplane $\mathbf{a}$, $\left\{\mathbf{a}, \mathbf{a}_{1}, \ldots, \mathbf{a}_{p}\right\}$ are in general position.

REMARK 3. If (3.1) holds for an unbounded sequence $\left\{r_{m}\right\}$, then we say that the ray is a $T$-direction for $\left\{r_{m}\right\}$. It is easy to see that a $T$-direction for $\left\{r_{m}\right\}$ is a $T$-direction.

A $T$-direction must be a Julia direction and a Borel direction of lower order. However, a $T$-direction may not be a Borel direction of the order and a Borel direction may not be a $T$ direction. This was proved by Zhang [28] for the case of meromorphic functions. Eremenko [6] proved the existence of Julia directions of a holomorphic curve.

THEOREM 3.1. If the Reisz measure $\mu$ corresponding to the holomorphic curve $f$ on the complex plane satisfies

$$
\limsup _{m \rightarrow \infty} \mu\left\{z: 2^{m} \leq|z| \leq 2^{m+1}\right\}=\infty,
$$

then $f$ has at least one Julia direction.

We remark on condition (3.3). From (3.3) it follows that

$$
\limsup _{r \rightarrow \infty} \frac{\mathcal{A}(r, f)}{\log r}=\infty \text {. }
$$

This implies that

$$
\limsup _{r \rightarrow \infty} \frac{\mathcal{T}(r, f)}{(\log r)^{2}}=\infty .
$$


In fact, suppose that $\mathcal{T}(r, f) \leq K(\log r)^{2}$ for a positive constant $K$, and then

$$
\mathcal{A}(r, f)=r \mathcal{T}^{\prime}(r, f) \leq \hat{K} \log r
$$

for suitable constant $\hat{K}$. A contradiction is derived. Also, it is easy to see that we can deduce (3.3) from (3.4).

Now we establish the existence of singular directions of holomorphic curves.

TheOREm 3.2. Let $\left\{r_{m}\right\}$ be a sequence of positive numbers outside $E(f)$ tending to $\infty$ such that

$$
\lim _{n \rightarrow \infty} \frac{T\left(r_{m}, f\right)}{\left(\log r_{m}\right)^{2}}=\infty
$$

Then there exists a $T$-direction of $f$ for $\left\{r_{m}\right\}$.

Proof. Suppose on the contrary that $f$ has no $T$-directions for $\left\{r_{m}\right\}$. Then for every radial $\arg z=\theta$, we have an angle containing it such that (3.1) with $r=r_{m}$ does not hold for $2 n+1$ hyperplanes in general position. Thus, we can choose finitely many radials $\arg z=\theta_{j}(1 \leq j \leq p)$ and an $\varepsilon>0$ such that $\left\{Z_{\varepsilon}\left(\theta_{j}\right): 1 \leq\right.$ $j \leq p\}$ is a covering of $\mathbb{C} \backslash\{0\}$, and for $2 n+1$ hyperplanes $\mathbf{a}_{1}^{j}, \mathbf{a}_{2}^{j}, \ldots, \mathbf{a}_{2 n+1}^{j}$ in general position, we have

$$
\sum_{k=1}^{2 n+1} N_{Z_{2 \varepsilon}\left(\theta_{j}\right)}\left(r_{m}, \mathbf{a}_{k}^{j}, f\right)=o\left(T\left(r_{m}, f\right)\right) \quad(m \rightarrow \infty) .
$$

In view of Theorem 2.1, $\mathcal{T}\left(r_{m}, Z_{\varepsilon}\left(\theta_{j}\right), f\right)=o\left(T\left(r_{m}, f\right)\right)(m \rightarrow \infty)$. Since

$$
\mathcal{T}(r, f) \leq \sum_{j=1}^{p} \mathcal{T}\left(r, Z_{\varepsilon}\left(\theta_{j}\right), f\right) \leq p \mathcal{T}(r, f),
$$

we have $T\left(r_{m}, f\right)=o\left(T\left(r_{m}, f\right)\right)$, and a contradiction has been derived.

We remark that if (3.4) holds, then (3.5) holds for some sequence $\left\{r_{m}\right\}$ outside $E(f)$, and further, a $T$-direction exists.

Theorem 3.2 confirms the existence of Borel directions for a holomorphic curve $f$ of positive order $\lambda(f)$. In fact, we can have a sequence $\left\{r_{m}\right\}$ outside $E(f)$ such that

$$
\lim _{m \rightarrow \infty} \frac{\log T\left(r_{m}, f\right)}{\log r_{m}}=\lambda(f) .
$$

Then the $T$-direction of $f$ for this sequence $\left\{r_{m}\right\}$ must be a Borel direction of order $\lambda(f)$.

\section{Growth of Holomorphic Curves with Radially Distributed Hyperplanes}

Let $f: \mathbb{C} \rightarrow \mathcal{P}^{n}(\mathbb{C})$ be a holomorphic curve on $\mathbb{C}$, and $\mathbf{f}=\left(f_{0}, f_{1}, \ldots, f_{n}\right)$ be a reduced representation of $f$. Let $H$ be a hyperplane with a reduced representation a of $H$. Consider the counting function of the preimage of $H$ under $f$ : 
$N(r, \mathbf{a}, f)=N_{\mathbb{C}}(r, \mathbf{a}, f)$. Define

$$
\delta(\mathbf{a}, f)=1-\limsup _{r \rightarrow \infty} \frac{N(r, \mathbf{a}, f)}{T(r, f)},
$$

which is called the deficiency of $f$ with respect to $H$, and if $\delta(\mathbf{a}, f)>0$, then $H$ is called a deficient hyperplane of $f$. Under the existence of a deficient hyperplane of $f$, we consider the estimation of growth of the holomorphic curve $f$ in view of some radially distributed hyperplanes. Since the Cartan characteristic characterizes the growth of a holomorphic curve, we will first give an estimate for the Cartan characteristic, and then the estimate can be controlled in view of the counting functions of argument distribution of the preimages of some hyperplanes, so that we obtain the estimate of growth order of a holomorphic curve in view of some radially distributed hyperplanes.

We begin with notation. Since the Cartan characteristic $T(r, f)$ of a holomorphic curve is increasing and logarithmic convex, we can consider its Pólya peak sequence. A positive increasing unbounded sequence $\left\{r_{m}\right\}$ is a sequence of Pólya peak of order $\sigma$ of $T(r, f)$ if there exist sequences $\left\{r_{m}^{\prime}\right\},\left\{r_{m}^{\prime \prime}\right\}$, and $\left\{\varepsilon_{m}\right\}$ such that

(1) $r_{m}^{\prime} \rightarrow \infty, r_{m} / r_{m}^{\prime} \rightarrow \infty, r_{m}^{\prime \prime} / r_{m} \rightarrow \infty$, and $\varepsilon_{m} \rightarrow 0$ as $m \rightarrow \infty$.

(2) $T(t, f) \leq\left(1+\varepsilon_{m}\right)\left(t / r_{m}\right)^{\sigma} T\left(r_{m}, f\right), r_{m}^{\prime} \leq t \leq r_{m}^{\prime \prime}$.

The sequence $\left\{r_{m}\right\}$ is called a strong Pólya peak sequence if, in addition, we have

(3) $T(t, f) / t^{\sigma-\varepsilon_{m}^{\prime}} \leq K T\left(r_{m}, f\right) / r_{m}^{\sigma-\varepsilon_{m}^{\prime}}, 1 \leq t \leq r_{m}^{\prime \prime}$, for a sequence $\varepsilon_{m}^{\prime} \rightarrow 0$ as $m \rightarrow \infty$ and a positive constant $K$.

From (3) it follows easily that

$$
\liminf _{m \rightarrow \infty} \frac{\log T\left(r_{m}, f\right)}{\log r_{m}} \geq \sigma .
$$

If $T(r, f)$ has the lower order $\tau<\infty$ and order $0<\lambda \leq \infty$, then for a finite positive number $\sigma$ with $\tau \leq \sigma \leq \lambda$ and a set $E$ of positive numbers with finite logarithmic measure, there must be a sequence of strong Polya peak $\left\{r_{m}\right\}$ of order $\sigma$ of $T(r, f)$ outside $E$ (cf. Theorem 1.1.3 in [31]).

Consider $p$ pairs of real numbers $\left\{\alpha_{j}, \beta_{j}\right\}$ such that

$$
-\pi \leq \alpha_{1}<\beta_{1} \leq \alpha_{2}<\beta_{2} \leq \cdots \leq \alpha_{p}<\beta_{p} \leq \pi, \quad \alpha_{p+1}=\alpha_{1}+2 \pi,
$$

and by $D=D\left(\alpha_{1}, \beta_{1}, \ldots, \alpha_{p}, \beta_{p}\right)$ we denote the corresponding ray system $\arg z=\alpha_{j}, \beta_{j}(1 \leq j \leq p)$. For the system $D$, define

$$
\begin{aligned}
\omega & =\omega(D)=\max \left\{\omega_{j}=\frac{\pi}{\beta_{j}-\alpha_{j}}: 1 \leq j \leq p\right\}, \\
\omega^{\prime} & =\omega^{\prime}(D)=\max \left\{\frac{\pi}{\alpha_{j+1}-\beta_{j}}: 1 \leq j \leq p\right\},
\end{aligned}
$$

and

$$
W(r, D, \mathbf{a}, f)=\max \left\{r^{\omega_{j}} B_{\alpha_{j}, \beta_{j}}(r, \mathbf{a}, f): 1 \leq j \leq p\right\}
$$


We estimate the Cartan characteristic $T(r, f)$ in terms of this $W$. This is our first result in this section.

THEOREM 4.1. Let $f: \mathbb{C} \rightarrow \mathcal{P}^{n}(\mathbb{C})$ be a holomorphic curve on $\mathbb{C}$ of lower order $\tau<\infty$ and order $0<\lambda \leq \infty$. Let $H$ be a hyperplane with a reduced representation $\mathbf{a}$ and $\delta=\delta(\mathbf{a}, f)>0$. Assume that for a $\sigma>0$ with $\tau \leq \sigma \leq \lambda$,

$$
\sum_{j=1}^{p}\left(\alpha_{j+1}-\beta_{j}\right)<\frac{4}{\sigma} \arcsin \sqrt{\frac{\delta}{2}} .
$$

Then for any sequence of Pólya peaks $\left\{r_{m}\right\}$ of order $\sigma$, we have

$$
T\left(r_{m}, f\right) \leq K W\left(r_{m}, D, \mathbf{a}, f\right)
$$

for some positive number $K$ only depending on $\left\{r_{m}\right\}$ independent of $m$.

In order to prove Theorem 4.1, we need the spread relation for holomorphic curves proved by Niino [17], which is an extension of Baernstein's spread relation for a meromorphic function. For a positive function $\Lambda(r)$ with $\Lambda(r) \rightarrow 0(r \rightarrow \infty)$, define

$$
E_{\Lambda}(r, H)=\left\{\theta \in(-\pi, \pi): \lambda_{H}\left(f\left(r e^{i \theta}\right)\right)>\Lambda(r) T(r, f)\right\},
$$

where $\lambda_{H}(f)$ is the Weil function of $f$ with respect to $H$.

Lemma 4.1. Let $f$ be a holomorphic curve on $\mathbb{C}$, and let $H$ be a deficient hyperplane with a reduced representation a and $\delta=\delta(\mathbf{a}, f)>0$. Then for any sequence of Pólya peaks $\left\{r_{m}\right\}$ of order $\sigma>0$ for $f$ and any positive function $\Lambda(r)$ with $\Lambda(r) \rightarrow 0(r \rightarrow \infty)$, we have

$$
\liminf _{r \rightarrow \infty} \operatorname{meas} E_{\Lambda}\left(r_{m}, H\right) \geq \min \left\{2 \pi, \frac{4}{\sigma} \arcsin \sqrt{\frac{\delta}{2}}\right\} .
$$

Proof of Theorem 4.1. Suppose that (4.2) does not hold. Then there exists a subsequence of $\left\{r_{m}\right\}$, denoted still by $\left\{r_{m}\right\}$, such that $W\left(r_{m}, D, \mathbf{a}, f\right)=o\left(T\left(r_{m}, f\right)\right)$. Set

$$
\Gamma(r)=\frac{W\left(r_{m}, D, \mathbf{a}, f\right)}{T\left(r_{m}, f\right)}, \quad r_{m-1}<r \leq r_{m},
$$

and $\Lambda(r)=\sqrt{\Gamma(r)}$. Obviously, $\Lambda(r) \rightarrow 0(r \rightarrow \infty)$. Taking an $\varepsilon>0$ such that

$$
\sum_{j=1}^{p}\left(\alpha_{j+1}-\beta_{j}+2 \varepsilon\right)+2 \varepsilon<\frac{4}{\sigma+2 \varepsilon} \arcsin \sqrt{\frac{\delta}{2}},
$$

in view of Lemma 4.1, we can find a $j_{0}$ such that

$$
\operatorname{meas}\left(E_{\Lambda}\left(r_{m}, H\right) \cap\left(\alpha_{j_{0}}+\varepsilon, \beta_{j_{0}}-\varepsilon\right)\right) \geq \frac{\varepsilon}{p}
$$

for a subsequence of $\left\{r_{m}\right\}$, denoted still by $\left\{r_{m}\right\}$. Then

$$
\int_{\alpha_{j_{0}}+\varepsilon}^{\beta_{j_{0}}-\varepsilon} \lambda_{H}\left(f\left(r_{m} e^{i \theta}\right)\right) \mathrm{d} \theta \geq \frac{\varepsilon}{p} \Lambda\left(r_{m}\right) T\left(r_{m}, f\right) .
$$


Thus,

$$
\begin{aligned}
W\left(r_{m}, D, \mathbf{a}, f\right) & \geq r_{m}^{\omega_{j_{0}}} B_{\alpha_{j_{0}}, \beta_{j_{0}}}\left(r_{m}, \mathbf{a}, f\right) \\
& =\frac{2 \omega_{j_{0}}}{\pi} \int_{\alpha_{j_{0}}}^{\beta_{j_{0}}} \lambda_{H}\left(f\left(r_{m} e^{i \theta}\right)\right) \sin \omega_{j_{0}}\left(\theta-\alpha_{j_{0}}\right) \mathrm{d} \theta \\
& \geq \frac{2 \omega_{j_{0}} \sin \omega_{j_{0}} \varepsilon}{\pi} \int_{\alpha_{j_{0}}+\varepsilon}^{\beta_{j_{0}}-\varepsilon} \lambda_{H}\left(f\left(r_{m} e^{i \theta}\right)\right) \mathrm{d} \theta \\
& \geq \frac{2 \omega_{j_{0}} \varepsilon \sin \omega_{j_{0}} \varepsilon}{\pi p} \Lambda\left(r_{m}\right) T\left(r_{m}, f\right) .
\end{aligned}
$$

On the other hand, we have

$$
W\left(r_{m}, D, \mathbf{a}, f\right)=\Lambda\left(r_{m}\right)^{2} T\left(r_{m}, f\right) .
$$

Thus, it follows that

$$
0<\frac{2 \omega_{j_{0}} \varepsilon \sin \omega_{j_{0}} \varepsilon}{\pi p} \leq \Lambda\left(r_{m}\right) \rightarrow 0 \quad(m \rightarrow \infty) .
$$

A contradiction is derived.

Now we control the Cartan characteristic in terms of the counting functions in some angular domains. We want to realize it by controlling the quantity $W$ in Theorem 4.1 in terms of the counting functions in some angular domains. To this end, we need the following lemmas.

Lemma 4.2. Let $f: \bar{\Omega}(\alpha, \beta) \rightarrow \mathcal{P}^{n}(\mathbb{C})$ be an $m$-nondegenerate holomorphic curve, and let $\mathbf{a}, \mathbf{a}_{0}, \mathbf{a}_{1}, \ldots, \mathbf{a}_{q}$ with $q=2 n-m$ be hyperplanes in general position. Then

$$
B_{\alpha, \beta}(r, \mathbf{a}, f) \leq m \sum_{j=0}^{q} \bar{C}_{\alpha, \beta}\left(r, \mathbf{a}_{j}, f\right)+R_{\alpha, \beta}(r, f) .
$$

Proof. In terms of Theorem 2.4 with the truncated form, we have

$$
\begin{aligned}
S_{\alpha, \beta}(r, f) & \leq C_{\alpha, \beta}(r, \mathbf{a}, f)+\sum_{k=0}^{q} C_{\alpha, \beta}^{m)}\left(r, \mathbf{a}_{k}, f\right)+R_{\alpha, \beta}(r, f) \\
& \leq C_{\alpha, \beta}(r, \mathbf{a}, f)+m \sum_{k=0}^{q} \bar{C}_{\alpha, \beta}\left(r, \mathbf{a}_{k}, f\right)+R_{\alpha, \beta}(r, f) .
\end{aligned}
$$

By Theorem 2.2 we have

$$
A_{\alpha, \beta}(r, \mathbf{a}, f)+B_{\alpha, \beta}(r, \mathbf{a}, f)+C_{\alpha, \beta}(r, \mathbf{a}, f)=S_{\alpha, \beta}(r, f)+O(1) .
$$

Combining the previous two inequalities yields the desired inequality by noting that $A_{\alpha, \beta}(r, \mathbf{a}, f) \geq 0$. 
We compare $C_{\alpha, \beta}$ to $N_{\alpha, \beta}$. The following is a version of Lemma 2.2.2 in [31] for a holomorphic curve.

Lemma 4.3. Let $f$ be a holomorphic curve on $\bar{\Omega}(\alpha, \beta)$, and a be a hyperplane. Then

$$
r^{\omega} C_{\alpha, \beta}(r, \mathbf{a}, f) \leq 4 \omega N(r)+2 \omega^{2} r^{\omega} \int_{1}^{r} \frac{N(t)}{t^{1+\omega}} \mathrm{d} t,
$$

where $N(t)=N_{\alpha, \beta}(t, \mathbf{a}, f)$ and $\omega=\frac{\pi}{\beta-\alpha}$. The inequality also holds with $\bar{C}$ and $\bar{N}$ instead of $C$ and $N$.

Proof. In terms of the definition of $C_{\alpha, \beta}(r, \mathbf{a}, f)$, we have

$$
\begin{aligned}
C_{\alpha, \beta}(r, \mathbf{a}, f) & \leq 2 \sum_{1 \leq\left|a_{n}\right| \leq r}\left(\frac{1}{\left|a_{n}\right|^{\omega}}-\frac{\left|a_{n}\right|^{\omega}}{r^{2 \omega}}\right) \\
& =2 \int_{1}^{r}\left(\frac{1}{t^{\omega}}-\frac{t^{\omega}}{r^{2 \omega}}\right) \mathrm{d} n(t) \\
& =2 \omega \int_{1}^{r} n(t)\left(\frac{1}{t^{\omega+1}}+\frac{t^{\omega-1}}{r^{2 \omega}}\right) \mathrm{d} t \\
& =2 \omega \int_{1}^{r}\left(\frac{1}{t^{\omega}}+\frac{t^{\omega}}{r^{2 \omega}}\right) \mathrm{d} N(t) \\
& \leq 4 \omega \frac{N(r)}{r^{\omega}}+2 \omega^{2} \int_{1}^{r} \frac{N(t)}{t^{\omega+1}} \mathrm{~d} t .
\end{aligned}
$$

Now we establish the second result in this section.

THEOREM 4.2. Let $f: \mathbb{C} \rightarrow \mathcal{P}^{n}(\mathbb{C})$ be an $m$-nondegenerate holomorphic curve on $\mathbb{C}$ of finite lower order $\tau$. Let $\mathbf{a}, \mathbf{a}_{0}, \mathbf{a}_{1}, \ldots, \mathbf{a}_{q}$ with $q=2 n-m$ be $q+2$ hyperplanes in general position, and $\delta=\delta(\mathbf{a}, f)>0$. Assume that for a ray system $D\left(\alpha_{1}, \beta_{1}, \ldots, \alpha_{p}, \beta_{p}\right),(4.1)$ holds with $\sigma=\max \{\omega(D), \tau\}$ and

$$
\sum_{k=0}^{q} N_{\alpha_{j}, \beta_{j}}\left(r, \mathbf{a}_{k}, f\right)=o(T(r, f)), \quad 1 \leq j \leq p .
$$

Then $\lambda(f) \leq \omega(D)$.

Proof. Suppose that $\lambda(f)>\omega(D)$. Then $\lambda(f)>\sigma$. Take an $\varepsilon>0$ such that $\lambda(f)>\sigma+\varepsilon$ and (4.1) holds for $\sigma+\varepsilon$. There exists a strong Pólya peak sequence $\left\{r_{m}\right\}$ of order $\sigma+\varepsilon$. In view of Theorem 4.1, we have (4.2) for some constant $K$.

We estimate $W\left(r_{m}, D, \mathbf{a}, f\right)$. It follows from Lemma 4.2 and Lemma 4.3 that

$$
\begin{aligned}
W\left(r_{m}, D, \mathbf{a}, f\right) \leq & C \max _{1 \leq j \leq p}\left\{N_{\alpha_{j}, \beta_{j}}\left(r_{m}\right)+r_{m}^{\omega_{j}} \int_{1}^{r_{m}} \frac{N_{\alpha_{j}, \beta_{j}}(t)}{t^{\omega_{j}+1}} \mathrm{~d} t\right\} \\
& +O\left(r_{m}^{\omega(D)} \log r_{m} T\left(r_{m}, f\right)\right),
\end{aligned}
$$


where $N_{\alpha_{j}, \beta_{j}}(t)=\sum_{k=0}^{q} N_{\alpha_{j}, \beta_{j}}\left(t, \mathbf{a}_{k}, f\right)$. Since

$$
\begin{aligned}
\int_{1}^{r_{m}} \frac{T(t, f)}{t^{\omega_{j}+1}} \mathrm{~d} t & \leq \int_{1}^{r_{m}}\left(\frac{t}{r_{m}}\right)^{\sigma+\varepsilon-\varepsilon_{m}^{\prime}} \frac{T\left(r_{m}, f\right)}{t^{\omega_{j}+1}} \mathrm{~d} t \\
& =\frac{T\left(r_{m}, f\right)}{r_{m}^{\sigma+\varepsilon-\varepsilon_{m}^{\prime}}} \int_{1}^{r_{m}} t^{\sigma+\varepsilon-\varepsilon_{m}^{\prime}-\omega_{j}-1} \mathrm{~d} t \\
& =\frac{1}{\sigma+\varepsilon-\varepsilon_{m}^{\prime}-\omega_{j}} \frac{T\left(r_{m}, f\right)}{r_{m}^{\omega_{j}}},
\end{aligned}
$$

we have

$$
r_{m}^{\omega_{j}} \int_{1}^{r_{m}} \frac{N_{\alpha_{j}, \beta_{j}}(t)}{t^{\omega_{j}+1}} \mathrm{~d} t=o\left(r_{m}^{\omega_{j}} \int_{1}^{r_{m}} \frac{T(t, f)}{t^{\omega_{j}+1}} \mathrm{~d} t\right)=o\left(T\left(r_{m}, f\right)\right) .
$$

Therefore, combining the previous inequalities yields

$$
W\left(r_{m}, D, \mathbf{a}, f\right)=o\left(T\left(r_{m}, f\right)\right)+O\left(r_{m}^{\omega(D)} \log r_{m} T\left(r_{m}, f\right)\right),
$$

and this, together with (4.2), implies that

$$
T\left(r_{m}, f\right)=O\left(r_{m}^{\omega(D)} \log r_{m} T\left(r_{m}, f\right)\right),
$$

so that

$$
\sigma+\varepsilon \leq \limsup _{m \rightarrow \infty} \frac{\log T\left(r_{m}, f\right)}{\log r_{m}} \leq \omega(D) \leq \sigma .
$$

A contradiction is derived.

REMARK 4. From Theorem 4.2 it follows that for a ray system $D\left(\alpha_{1}, \ldots, \alpha_{q}\right)$, if for any small $\varepsilon>0$,

$$
\sum_{k=0}^{q} N_{\alpha_{j}+\varepsilon, \alpha_{j+1}-\varepsilon}\left(r, \mathbf{a}_{k}, f\right)=o(T(r, f)), \quad 1 \leq j \leq p,
$$

and $\lambda(f)>\omega(D)=\max \left\{\pi /\left(\alpha_{j+1}-\alpha_{j}\right): 1 \leq j \leq p\right\}$, then every deficient hyperplane (if exists) of $f$ is in the linear span of $\mathbf{a}_{k}, 0 \leq k \leq q$, and furthermore, $f$ has at most $n+1$ deficient hyperplanes in general position.

\section{Applications of Tsuji Characteristic of Holomorphic Curves on an Angle}

With the help of the Tsuji characteristic of holomorphic curves for an angle, some discussions on the complex plane can be transferred to those on an angle. The reader is referred to Gundersen and Hayman [13] and $\mathrm{Ru}$ [19] for the corresponding results on the complex plane to those on an angle in this section and for the related references.

We say that a holomorphic curve $f$ on an angle $\Omega$ is transcendental in the sense of Tsuji if

$$
\limsup _{r \rightarrow \infty} \frac{\mathfrak{T}_{\Omega}(r, f)}{\log r}=\infty
$$


The following is a modification of the version of the Borel theorem stated in [31], which can be proved by Theorem 2.5.

THEOREM 5.1. Let $f_{j}(z)(j=1,2, \ldots, q)$ be transcendental meromorphic functions in an angle $\Omega=\{\alpha<\theta<\beta\}$ with $0<\alpha<\beta \leq 2 \pi$. Assume that $f_{j}(z) / f_{i}(z)$ is not a constant for $i \neq j$ and

$$
\sum_{j=1}^{q}\left(\overline{\mathfrak{N}}_{\alpha, \beta}\left(r, f_{j}\right)+\overline{\mathfrak{N}}_{\alpha, \beta}\left(r, \frac{1}{f_{j}}\right)\right)=o\left(\mathfrak{T}_{\alpha, \beta}(r)\right),
$$

where $\mathfrak{T}_{\alpha, \beta}(r)=\min \left\{\mathfrak{T}_{\alpha, \beta}\left(r, f_{j} / f_{i}\right): 1 \leq j<i \leq q\right\}$. Then $\left\{f_{j}(z): j=1,2\right.$, $\ldots, q\}$ is linearly independent.

In view of Theorem 2.5, we can discuss in an angle the ABC problem for analytic functions, Fermat-type equation for meromorphic functions, and Waring's problem for analytic functions. We also establish a uniqueness theorem of holomorphic curves in an angle. Following the work of Stoll [22] on the complex plane, we can establish the following:

THEOREM 5.2. Let $f_{j}(z)(j=1,2, \ldots, q)$ be linearly nondegenerate transcendental holomorphic curves with reduced representation $\mathbf{f}_{j}$ from an angle $\Omega(\alpha, \beta)$ to $\mathcal{P}^{n}(\mathbb{C})$. Let $H_{i}(i=1,2, \ldots, p)$ be hyperplanes on $\mathcal{P}^{n}(\mathbb{C})$ in general position. Assume that $A_{j}=f_{1}\left(H_{j}\right)=\cdots=f_{q}\left(H_{j}\right)(j=1,2, \ldots, p)$ and $A_{i} \cap A_{j}=\emptyset$ for $i \neq j$. Let $s, 2 \leq s \leq q$, be an integer such that for any $1 \leq j_{1}<j_{2}<\cdots<j_{s} \leq q$, we have $\mathbf{f}_{j_{1}}(z) \wedge \mathbf{f}_{j_{2}}(z) \wedge \cdots \wedge \mathbf{f}_{j_{s}}(z)=0, z \in \bigcup_{j=1}^{p} A_{j}$. If $p>\frac{q n}{q-s+1}+n+1$, then we have

$$
\mathbf{f}_{1}(z) \wedge \mathbf{f}_{2}(z) \wedge \cdots \wedge \mathbf{f}_{q}(z) \equiv 0, \quad z \in \Omega
$$

Stoll's result for the complex plane was extended by $\mathrm{Ru}$ [20] to moving targets. The same method can realize the extension of Theorem 5.2 to moving targets. The reader is referred to [5] and [3] for further development of uniqueness theorems on the complex plane.

\section{Argument Distribution of Algebroid Functions}

We investigate the algebroid functions from the point of view of holomorphic curves. A $v$-valued algebroid function on $\bar{\Omega}(\alpha, \beta)$ is the solution of the algebraic equation

$$
\psi(z, w)=A_{v}(z) w^{v}+A_{v-1}(z) w^{v-1}+\cdots+A_{0}(z)=0,
$$

where $A_{j}(z), 0 \leq j \leq v$, is an analytic function without common zeros on $\bar{\Omega}$, and $\psi(z, w)$ is irreducible in $w$. Therefore, $f_{w}=\left[A_{v}: \ldots: A_{1}: A_{0}\right]$ is a holomorphic curve from $\bar{\Omega}$ to $\mathcal{P}^{v}(\mathbb{C})$, and $\mathbf{f}_{w}=\left(A_{v}, A_{v-1}, \ldots, A_{1}, A_{0}\right)$ is a reduced representation of $f_{w}$.

Associated with a $v$-valued algebroid function is a Riemann surface $\mathfrak{R}_{w}$ on which the algebroid function is single valued. All of singular points produced 
by the irreducible equation are algebraic, and so in a neighborhood of any point on $\Omega, w(z)$ has an expansion of the Puiseux series. Thus, we can write the branch functions $w_{1}(z), \ldots, w_{v}(z)$ of $w(z)$. A point $z_{0}$ is a branch point of $w(z)$ if and only if $w_{i}\left(z_{0}\right)=w_{j}\left(z_{0}\right)$ for some pair $i \neq j$.

Therefore, there exist two equivalent formats in defining the characteristic, counting function and proximity function of an algebroid function: one is in view of the branch functions, the other is in view of a holomorphic curve, but they are equivalent, which is proven in the following lemma. Set, for a complex number $a$,

$$
\begin{aligned}
\log ^{+} \frac{1}{|w(z)-a|} & =\sum_{k=1}^{v} \log ^{+} \frac{1}{\left|w_{k}(z)-a\right|}, \\
\log ^{+}|w(z)| & =\sum_{k=1}^{v} \log ^{+}\left|w_{k}(z)\right|,
\end{aligned}
$$

and $\vec{a}=\left(a^{v}, a^{v-1}, \ldots, a, 1\right)$ and $\vec{\infty}=(1,0, \ldots, 0)$. Therefore, to a hyperplane in $\mathcal{P}^{v}(\mathbb{C})$, there corresponds any value in $\hat{\mathbb{C}}$.

Lemma 6.1. Let $w$ be the algebroid function defined by (6.1). Then for $a \in \mathbb{C}$, we have

$$
\log ^{+} \frac{1}{|w(z)-a|}=\lambda_{\vec{a}}\left(f_{w}(z)\right)+\varepsilon(z, a)
$$

and

$$
\log ^{+}|w(z)|=\log \left\|\mathbf{f}_{w}(z)\right\|-\log \left|A_{v}(z)\right|+\varepsilon(z),
$$

where

$$
|\varepsilon(z, a)| \leq 6 v \log 3(1+|a|)+v^{3}, \quad|\varepsilon(z)| \leq v^{3} .
$$

Lemma 6.1 was proved in [25] and [18].

Define the characteristic, counting function, and proximity function of Nevanlinna and Tsuji of a $v$-valued algebroid function $w(z)$ on $\Omega(\alpha, \beta)$ as follows:

$$
\begin{aligned}
A_{\Omega}(r, a, w)= & \frac{\omega}{\pi} \int_{1}^{r}\left(\frac{1}{t^{\omega}}-\frac{t^{\omega}}{r^{2 \omega}}\right) \\
& \times\left(\log ^{+} \frac{1}{\left|w\left(t e^{i \alpha}\right)-a\right|}+\log ^{+} \frac{1}{\left|w\left(t e^{i \beta}\right)-a\right|}\right) \frac{\mathrm{d} t}{t}, \\
B_{\Omega}(r, a, w)= & \frac{2 \omega}{\pi r^{\omega}} \int_{\alpha}^{\beta} \log ^{+} \frac{1}{\left|w\left(r e^{i \theta}\right)-a\right|} \sin \omega(\theta-\alpha) \mathrm{d} \theta, \\
C_{\Omega}(r, a, w)= & \frac{1}{2 \pi} \int_{1}^{r} \int_{\alpha}^{\beta}\left(\frac{1}{t^{\omega}}-\frac{t^{\omega}}{r^{2 \omega}}\right) \sin \omega(\theta-\alpha) \Delta v_{\vec{a}}\left(t e^{i \theta}\right) \\
= & \sum_{k}\left(\frac{1}{r_{k}^{\omega}}-\frac{r_{k}^{\omega}}{r^{2 \omega}}\right) \sin \omega\left(\theta_{k}-\alpha\right),
\end{aligned}
$$


where $z_{k}=r_{k} e^{i \theta_{k}}$ is a zero of $\left\langle\mathbf{f}_{w}, \vec{a}\right\rangle$ on $\Omega(r)$, counted with multiplicities, $v_{\vec{a}}=\log \left|\left\langle\mathbf{f}_{w}, \vec{a}\right\rangle\right|$, and

$$
\begin{aligned}
S_{\Omega}(r, a, w) & =A_{\Omega}(r, a, w)+B_{\Omega}(r, a, w)+C_{\Omega}(r, a, w) \\
\mathfrak{M}_{\Xi}(r, a, w) & =\frac{1}{2 \pi} \int_{\arcsin \left(r^{-\omega}\right)}^{\pi-\arcsin \left(r^{-\omega}\right)} \log ^{+} \frac{1}{\left|w\left(r e^{i\left(\alpha+\omega^{-1} \theta\right)} \sin ^{\omega^{-1}} \theta\right)-a\right|} \frac{\mathrm{d} \theta}{r^{\omega} \sin ^{2} \theta}, \\
\mathfrak{N}_{\Xi}(r, a, w) & =\frac{1}{2 \pi} \int_{0}^{\pi} \int_{1}^{r(\sin \omega(\theta-\alpha))^{\omega^{-1}}}\left(\frac{\sin \omega(\theta-\alpha)}{t^{\omega}}-\frac{1}{r^{\omega}}\right) \Delta v_{\vec{a}}\left(t e^{i \theta}\right) \\
& =\sum_{k}\left(\frac{\sin \omega\left(\theta_{k}-\alpha\right)}{r_{k}^{\omega}}-\frac{1}{r^{\omega}}\right)
\end{aligned}
$$

where $z_{k}=r_{k} e^{i \theta_{k}}$ is a zero of $\left\langle\mathbf{f}_{w}, \vec{a}\right\rangle$ on $\Xi(r)$, counted with multiplicities, and

$$
\mathfrak{T}_{\Xi}(r, a, w)=\mathfrak{M}_{\Xi}(r, a, w)+\mathfrak{N}_{\Xi}(r, a, w) .
$$

To make a more careful discussion of algebroid functions, we classify algebroid functions with the coefficients of the algebraic equation (6.1). We say that the algebroid function defined by (6.1) is $m$-nondegenerated if the holomorphic curve $f_{w}$ is $m$-nondegenerated. In terms of Theorem 2.4, Theorem 2.5, and Lemma 6.1, we can establish the second main theorems of algebroid functions in an angle for the Nevanlinna and Tsuji characteristics.

THEOREM 6.1. Let $w$ be an $m$-nondegenerate $v$-valued algebroid function on $\bar{\Omega}(\alpha-\varepsilon, \beta+\varepsilon)$ for some $\varepsilon>0$, and $a_{1}, a_{2}, \ldots, a_{q}$ be $q$ distinct values on $\hat{\mathbb{C}}$. Then we have

$$
\begin{aligned}
& (q-2 v+m-1) S_{\alpha, \beta}(r, w) \\
& \quad \leq \sum_{k=1}^{q} C_{\alpha, \beta}\left(r, a_{k}, w\right)-C_{\alpha, \beta}(r, 0, W)+R_{\alpha, \beta}(r, w),
\end{aligned}
$$

where $W$ is the Wronskian of $m+1$ linearly independent elements of $A_{0}, A_{1}, \ldots$, $A_{v}$, and $R_{\alpha, \beta}(r, w)$ is the error term with the estimate

$$
R_{\alpha, \beta}(r, w) \leq K\left(\log ^{+} S_{\alpha-\varepsilon, \beta+\varepsilon}(r, w)+\log ^{+} r+1\right),
$$

for all $r>1$, except possibly a set of finite linear measure, where $K$ is a constant depending on $\varepsilon$.

We leave the statement of the second main theorem of algebroid functions in an angle for Tsuji's characteristic to the reader. Thus, Theorem 4.1, Theorem 4.2, and Theorem 5.2 can be transferred to produce the corresponding results for an $m$-nondegenerate algebroid function.

Finally, let us give a version of the second main theorem of algebroid functions for Ahlfors-Shimizu characteristic in an angle corresponding to Theorem 2.1. Let $w$ be the $v$-valued algebroid function defined by (6.1), and $w_{j}(1 \leq j \leq v)$ be its 
branch functions. Define

$$
\mathcal{A}(r, \Omega, w)=\sum_{j=1}^{v} \frac{1}{\pi} \iint_{\Omega(r)} \frac{\left|w_{j}^{\prime}(z)\right|^{2}}{\left(1+\left|w_{j}(z)\right|^{2}\right)^{2}} \mathrm{~d} \sigma(z)
$$

and

$$
\mathcal{T}(r, \Omega, w)=\int_{1}^{r} \frac{\mathcal{A}(t, \Omega, w)}{t} \mathrm{~d} t .
$$

Theorem 6.2. Let $w$ be the $v$-valued algebroid function defined by (6.1) on $\mathbb{C}$, and $a_{1}, a_{2}, \ldots, a_{q}$ be $q$ distinct values on $\hat{\mathbb{C}}$. Then for an angular domain $\Omega(\alpha, \beta)$ and arbitrary $\epsilon>0$ such that $\alpha+\epsilon<\beta-\epsilon$, we have

$$
\begin{gathered}
(q-2 n) \mathcal{A}\left(r, \Omega_{\epsilon}, w\right) \leq \sum_{\substack{k=1\\
}}^{q} n_{\Omega}\left(r, a_{k}, w\right)+o(\mathcal{A}(r, \Omega, w)) \\
+O(\log r)+\hat{\mu}\left(\Omega_{\epsilon}(r)\right)
\end{gathered}
$$

and

$$
\begin{aligned}
(q-2 n) \mathcal{T}\left(r, \Omega_{\epsilon}, w\right) \leq & \sum_{k=1}^{q} N_{\Omega}\left(r, a_{k}, w\right)+o(\mathcal{T}(r, \Omega, w)) \\
& +O(\log r)^{2}+\int_{1}^{r} \frac{\hat{\mu}\left(\Omega_{\epsilon}(t)\right)}{t} \mathrm{~d} t
\end{aligned}
$$

for $r \notin E$, where $E$ has a finite logarithmic measure, and $\hat{\mu}$ is Riesz charge of a $\delta$-subharmonic function $\varepsilon(z)$ with $|\varepsilon(z)| \leq v^{3}+\log \sqrt{2}$ such that $\hat{\mu}(\{|z|<r\})=$ $O(1)$.

Proof. We only prove (6.3). Noting that for any complex number $a, \log ^{+}|a| \leq$ $\log \sqrt{1+|a|^{2}} \leq \log ^{+}|a|+\log \sqrt{2}$, in view of Lemma 6.1 we have

$$
\sum_{j=1}^{v} \log \sqrt{1+\left|w_{j}(z)\right|^{2}}=\log \left\|\mathbf{f}_{w}(z)\right\|-\log \left|A_{v}(z)\right|+\varepsilon(z),
$$

where $|\varepsilon(z)| \leq v^{3}+\sqrt{2}$. It is clear that $\varepsilon(z)$ is $\delta$-subharmonic on $\mathbb{C}$. Let $\hat{\mu}$ be the Riesz charge of $\varepsilon(z)$, and then $\hat{\mu}(\{|z|<r\})=O(1)$. It follows from (6.4) that

$$
\begin{aligned}
\frac{1}{2 \pi} \sum_{j=1}^{v} \iint_{\Omega_{\epsilon}(r)} \Delta \log \sqrt{1+\left|w_{j}(z)\right|^{2}} \\
=\frac{1}{2 \pi} \iint_{\Omega_{\epsilon}(r)} \Delta \log \left\|\mathbf{f}_{w}(z)\right\| \\
\quad-\frac{1}{2 \pi} \iint_{\Omega_{\epsilon}(r)} \Delta \log \left|A_{v}(z)\right|+\frac{1}{2 \pi} \iint_{\Omega_{\epsilon}(r)} \Delta \varepsilon(z) \\
=\frac{1}{2 \pi} \iint_{\Omega_{\epsilon}(r)} \Delta \log \left\|\mathbf{f}_{w}(z)\right\|-n_{\Omega}\left(r, \frac{1}{A_{v}}\right)+\frac{1}{2 \pi} \iint_{\Omega_{\epsilon}(r)} \Delta \varepsilon(z) .
\end{aligned}
$$


Using Theorem 2.1 to the holomorphic curve $f_{w}$, together with the last equality, yields (6.3).

We remark that the second fundamental inequality for the Ahlfors-Shimizu characteristic of algebroid functions in an angle were established in other references, for example, in [16], in terms of Ahlfors theory of covering surface, but in the inequality there is a term of the counting function of branch points that always brings the difficulty to application of the inequality. However, our second inequality has no this term. The existence of $T$-direction of algebroid functions can be immediately deduced by Theorem 6.2, which was established in [26] and [27].

AcKnOwLedgments. I would like to express my gratitude to Alex Eremenko for his valuable comments and indicating me Fedorov and Grishin's paper [9] and to $\mathrm{Ru}$ Min for his valuable suggestion in improving this paper.

\section{References}

[1] H. Cartan, Sur les zéros des combinaisons linéaires de p fonctions holomorphes données, Mathematica Cluj 7 (1933), 5-31.

[2] T. Ganelius, W. Hayman, and D. J. Newman, Lectures on approximation and value distribution; value distribution and exceptional sets, Séminaire de Mathématiques Supérieures, pp. 79-147, Les Presses de l'Université de Montréal, Montréal, 1982.

[3] G. Dethloff and T. V. Tan, A uniqueness theorem for meromorphic maps with moving hypersurfaces, arXiv:0902.0280v1.

[4] D. Drasin and W. Hayman, Value distribution of functions meromorphic in an angle, Proc. Lond. Math. Soc. (3) 48 (1984), no. 3, 319-340.

[5] M. Dulock and M. Ru, Uniqueness of holomorphic curves into Abelian varieties, Trans. Amer. Math. Soc. 363 (2011), 131-142.

[6] A. Eremenko, Julia directions for holomorphic curves, preprint in A. Eremenko's home pape.

[7] __ Value distribution and potential theory, Proceedings of the ICM, 2, pp. 681690, Higher Education Press, Beijing, 2002.

[8] A. Eremenko and M. L. Sodin, The value distribution of meromorphic functions and meromorphic curves from the point of view of potential theory, St. Petersburg Math. J. 3 (1992), 109-136.

[9] M. A. Fedorov and A. F. Grishin, Some questionss of Nevanlinna theory for the complex half plane, Math. Phys. Anal. Geom. 1 (1998), 223-271.

[10] E. V. Gleizer, Meromorphic functions with zeros and poles in small angles, Sib. Mat. Zh. 26 (1985), no. 4, 22-37, II, Sib. Mat. Zh. 31 (1990), no. 2, 9-20.

[11] A. A. Goldberg, Nevanlinna's lemma on the logarithmic derivative of meromorphic function, Mat. Zametki 17 (1975), no. 4, 525-529.

[12] A. A. Goldberg and I. V. Ostrovskii, Value distribution of meromorphic functions, Transl. Math. Monogr., 236, American Mathematical Society, Providence, RI, 2008.

[13] G. Gundersen and W. Hayman, The strength of Cartan's version of Nevanlinna theory, Bull. Lond. Math. Soc. 36 (2004), 433-454.

[14] W. K. Hayman, Waring's problem für analytische Funktionen, Bayer. Akad. Wiss. Math.-Natur. Kl. Sitzungsber. 1984 (1985), 1-13.

[15] W. Hayman and L. Yang, Growth and values of functiona regular in an angle, Proc. Lond. Math. Soc. (3) 44 (1982), no. 3, 193-214. 
[16] Q. Lu and Y. X. Gu, On Nevanlinna directions of algebroid functions, Acta Math. Sci. Ser. B Engl. Ed. 25 (2005), no. 2, 367-375.

[17] K. Niino, General defect relations of holomorphic curves, Trans. Amer. Math. Soc. 289 (1985), no. 1, 99-113.

[18] V. P. Petrenko, Growth and distribution of values of algebroid functions, Math. Zametki 26 (1979), no. 4, 513-522.

[19] M. Ru, Nevanlinna theory and its relation to Diophantine approximation, World Scientific, Singapore, New Jersey, London, Hong Kong, 2001.

[20] _ A uniqueness theorem with moving targets without counting multiplicity, Proc. Amer. Math. Soc. 129 (2001), no. 9, 2701-2707.

[21] M. Ru and W. Stoll, The second main theorem for moving targets, J. Geom. Anal. 1 (1991), 99-138.

[22] W. Stoll, On the propagation of dependences, Pacific J. Math. 139 (1989), 311-337.

[23] M. Tsuji, Potential theory in modern function theory, Maruzen Co. LTD, Tokyo, 1959.

[24] Z. H. Tu, On the Julia directions of the value distribution of holomorphic curves in $\mathcal{P}\left(\mathbb{C}^{n}\right)$, Kodai Math. J. 19 (1996), 1-6.

[25] G. Valiron, Sur la derivée des fonctions algébrö̈des, Bull. Sci. Math. 59 (1931), 1739.

[26] Z. J. Wu, On T direction of algebroid functions, J. Math. Kyoto Univ. 47 (2007), no. 4, 767-779.

[27] Z. X. Xuan, On the existence of $T$ direction of algebroid functions: a problem of J. $H$. Zheng, J. Math. Anal. Appl. 341 (2008), no. 1, 540-547.

[28] Q. D. Zhang, T directions and Borel directions of meromorphic functions with finite positive order, Acta Math. Sinica (Chin. Ser.) 50 (2007), no. 2, 413-420.

[29] X. L. Zhang, A fundamental inequality for meromorphic functions in an angular domain and its application, Acta Math. Sin, New Ser. 10 (1994), no. 3, 308-314.

[30] J. H. Zheng, On transcendental meromorphic functions with radially distributed values, Science in China, Series A. Math. 47 (2004), no. 3, 401-416.

[31] _ Value distribution of meromorphic functions, Springer-Verlag, Berlin Heidelberg, 2010.

Department of Mathematical

Sciences

Tsinghua University

Beijing, 100084

People's Republic of China 